\title{
EQUIVARIANT STABLE SHEAVES AND TORIC GIT
}

\author{
ANDREW CLARKE AND CARL TIPLER
}

\begin{abstract}
For $(X, L)$ a polarized toric variety and $G \subset \operatorname{Aut}(X, L)$ a torus, denote by $Y$ the GIT quotient $X / / G$. We define a family of fully faithful functors from the category of torus equivariant reflexive sheaves on $Y$ to the category of torus equivariant reflexive sheaves on $X$. We show, under a genericity assumption on $G$, that slope stability is preserved by these functors if and only if the pair $((X, L), G)$ satisfies a combinatorial criterion. As an application, when $(X, L)$ is a polarized toric orbifold of dimension $n$, we relate stable equivariant reflexive sheaves on certain $(n-1)$-dimensional weighted projective spaces to stable equivariant reflexive sheaves on $(X, L)$.
\end{abstract}

\section{INTRODUCTION}

The construction of moduli spaces of projective varieties and vector bundles is a fundamental problem in algebraic geometry. Given a polarized variety $(X, L)$ or a vector bundle $\mathcal{E}$ on $(X, L)$, one considers various stability notions for $X$ and $\mathcal{E}$ (see e.g. 1,5] for K-stability of varieties, and [10 for slope stability of bundles). In the presence of symmetries for $(X, L)$, that is, given an algebraic action of a reductive Lie group $G$ on $(X, L)$, it is natural to ask whether these stability notions persist on the GIT quotient $Y$ of $(X, L)$ by $G$. By the Yau-Tian-Donaldson conjecture [1,5] and the Kobayashi-Hitchin correspondence [13, the stability of $(X, L)$ or $\mathcal{E}$ can be related to the existence of a canonical metric on the underlying complex object, variety or bundle. From this differential geometrical point of view, $G$-orbits can detect curvature on $X$, and canonical metrics are not necessarily preserved under GIT quotients. As a motivating case, in [6, Futaki investigated GIT quotients of Fano varieties, giving a condition for the symplectic reduction of $X$ to be KählerEinstein. It is then natural to expect a relation between the stability of $X$, of the quotient $Y$, and the geometric properties of the representation $G \rightarrow \operatorname{Aut}(X, L)$. In this paper, we provide an example of such an interplay, by relating slope stability for reflexive sheaves on $X$ and $Y$ to a combinatorial criterion on the $G$-action, in the equivariant context of toric geometry (see also [7, 18, for related results).

A vector bundle, or more generally a torsion-free sheaf $\mathcal{E}$ on a complex projective variety $X$ is said to be slope stable with respect to an ample $\mathbb{R}$-divisor $\alpha \in N^{1}(X)_{\mathbb{R}}$ if for any subsheaf $\mathcal{F}$ with $0<\operatorname{rk} \mathcal{F}<\operatorname{rk} \mathcal{E}$, the slope inequality holds

$$
\mu_{\alpha}(\mathcal{F})<\mu_{\alpha}(E)
$$

where the slope is given by the intersection theoretical formula:

$$
\mu_{\alpha}(\mathcal{E})=\frac{c_{1}(\mathcal{E}) \cdot \alpha^{n-1}}{\operatorname{rk} \mathcal{E}} .
$$

Date: December 15, 2021. 
The notion of slope stability originated in the construction of moduli spaces of sheaves [10]. Assume now that $(X, L)$ is a polarized toric variety over $\mathbb{C}$, that is endowed with an effective action of a complex torus $T_{X}$ with open and dense orbit. We further assume the toric varieties that we consider to come from fans, and in particular to be normal. We denote by $N$ the lattice of one-parameter subgroups of $T_{X}$, so that $T_{X}=N \otimes_{\mathbb{Z}} \mathbb{C}^{*}$. Consider $G \subset T_{X}$ a subtorus, given by a sublattice $N_{0} \subset N$, that is $G=N_{0} \otimes_{\mathbb{Z}} \mathbb{C}^{*}$. For any linearization $\gamma: T_{X} \rightarrow \operatorname{Aut}(L)$, we can form a toric variety obtained by GIT quotient $Y=X / / G$. To avoid finite quotients, we will assume that $N_{0}$ is saturated in $N$, that is $N_{0}=N \cap\left(N_{0} \otimes \mathbb{R}\right)$. We will further assume that the restriction to $G$ of the linearization $\gamma$ on $L$ is generic, which is that the stable and semi-stable loci coincide (see Section 3.1). Under these hypothesis, we build a family of fully faithful functors

$$
\mathfrak{P}_{\mathbf{i}}: \mathfrak{R e f}^{T_{Y}}(Y) \rightarrow \mathfrak{R e f}^{T_{X}}(X)
$$

that embeds the category of torus equivariant reflexive sheaves 11 on $Y$ into the category of torus equivariant reflexive sheaves on $X$ (Section 3.3). Given an ample class $\alpha \in N^{1}(Y)_{\mathbb{R}}$ on $Y$, we will say that such a functor $\mathfrak{P}_{\mathbf{i}}$ preserves slope stability notions from $(Y, \alpha)$ to $(X, L)$ if an element $\mathcal{E} \in \mathfrak{R e f}^{T_{Y}}(Y)$ is slope stable (resp. semistable, polystable) with respect to $\alpha$ if and only if $\mathfrak{P}_{\mathbf{i}}(\mathcal{E})$ is slope stable (resp. semistable, polystable) with respect to $L$ (see Section 4.1 for the definition of these notions). Then our main result goes as follows:

Theorem 1.1. Let $(X, L)$ be a polarized toric variety with torus $T_{X}=N \otimes_{\mathbb{Z}} \mathbb{C}^{*}$. Let $G=N_{0} \otimes_{\mathbb{Z}} \mathbb{C}^{*}$ be a subtorus for a saturated sublattice $N_{0} \subset N$. Let $\gamma: T_{X} \rightarrow \operatorname{Aut}(L)$ be a generic linearization of $G$, and denote by $Y$ the associated GIT quotient $X / / G$. Then, the following statements are equivalent:

i) There exists an ample class $\alpha \in N^{1}(Y)_{\mathbb{R}}$ on $Y$ such that the functors $\mathfrak{P}_{\mathrm{i}}$ preserve slope stability notions from $(Y, \alpha)$ to $(X, L)$.

ii) The pair $((X, L),(G, \gamma))$ satisfies the Minkowski condition

$$
\sum_{D \subset X^{s}} \operatorname{deg}_{L}(D) u_{D}=0 \quad \bmod N_{0} \otimes_{\mathbb{Z}} \mathbb{R} .
$$

Moreover, there is at most one class $\alpha$ on $Y$ satisfying (i) up to scale.

In the statement of Theorem [1.1, the sum (1) is over the set of $T_{X}$-invariant irreducible divisors in the stable locus $X^{s}$ of the $G$-action and $u_{D}$ denotes the primitive generator of the ray associated to $D$ in the fan of $X$ (see Section 2).

The Minkowski condition (11) is a very restrictive condition on $(G, \gamma)$. We will say that a subtorus $G \subset T_{X}$ is compatible with $(X, L)$ if there is a generic linearization $\gamma: T_{X} \rightarrow \operatorname{Aut}(L)$ for $G$ such that $((X, L),(G, \gamma))$ satisfies the Minkowski condition. We obtain in Lemma 5.2 an explicit bound, depending on the dimension and the number of rays in the fan, on the number of compatible one-parameter subgroups for polarized projective toric varieties satisfying a mild hypothesis. Nevertheless, we manage to show the following:

Proposition 1.2. Let $(X, L)$ be a n-dimensional polarized toric orbifold. Denote by $m$ the number of torus fixed points of $X$. Up to replacing $L$ by a sufficiently high power, there are at least $m$ one-parameter subgroups of $T_{X}$ compatible with $(X, L)$

\footnotetext{
${ }^{1}$ Also known as toric reflexive sheaves in the literature.
} 
giving distinct GIT quotients. The associated GIT quotients for these subgroups are weighted projective spaces.

Remark 1.3. By a toric orbifold we mean a toric variety with isolated quotient singularities, corresponding to a simplicial fan, as in 2, Theorem 3.1.19 and Definition 3.1.18].

Compact weighted projective spaces are precisely the projective toric orbifolds of Picard rank 1, and as such are the simplest projective toric orbifolds. It is then interesting to be able to lift stable sheaves on these simpler objects to general toric orbifolds. We expect as an application of Theorem 1.1 and Proposition 1.2 to develop a new geometrical construction of stable bundles of low rank on toric projective varieties.

A fundamental theorem of Mehta and Ramanathan states that the restriction of a slope stable reflexive sheaf $\mathcal{E}$ on $X$ to a general complete intersection $Z \subset$ $X$ of sufficiently high degree is again slope stable [16]. To the knowledge of the authors, there is no similar general statement for projections $\pi: X \rightarrow Y$, and our construction provides a result in this direction. More precisely, from Theorem 1.1 we deduce:

Corollary 1.4. Let $Y$ be a projective toric variety, $\left(D_{i}\right)_{1 \leq i \leq r}$ invariant Cartier divisors on $Y$ and $V$ the decomposable toric vector bundle on $Y$ associated to the locally free sheaf

$$
\mathcal{F}=\mathcal{O}_{Y} \oplus \mathcal{O}_{Y}\left(D_{1}\right) \oplus \cdots \oplus \mathcal{O}_{Y}\left(D_{r}\right) .
$$

Consider the toric variety $X=\mathbb{P}\left(V^{\vee}\right)$, with projection map $\pi: X \rightarrow Y$. Let $L_{Y}$ be a polarization on $Y$ such that $L_{X}=\pi^{*} L_{Y} \otimes \mathcal{O}_{X}(1)$ is ample on $X$. Then, there exists a real ample class $\alpha \in N^{1}(Y)_{\mathbb{R}}$ such that an equivariant reflexive sheaf $\mathcal{E}$ on $(Y, \alpha)$ is slope stable if and only if $\pi^{*} \mathcal{E}$ is slope stable on $\left(X, L_{X}\right)$.

Remark 1.5. In the setting of Corollary 1.4 we give examples where we can determine the class $\alpha$ on $Y$ (see Section 5.3). This class is not the one obtained from the GIT quotient of $\left(X, L_{X}\right)$, that being $L_{Y}$ in this case. A quick look at the examples coming from Corollary 1.4 suggests that in most cases, $\alpha$ will be different from $L_{Y}$. It would be interesting to obtain a general formula for $\alpha$ in terms of the geometric data $(X, L)$ and $G$, and in particular to understand if $\alpha$ is always rational or not.

The proof of Theorem 1.1 is divided in two main parts. The first one, in Section 3, is the construction of the functors $\mathfrak{P}_{\mathbf{i}}$. It is naturally associated to the study of the descent of equivariant reflexive sheaves on a toric variety $X$ under a generic toric GIT quotient $X \rightarrow Y$. Let us denote by $\iota: X^{s} \rightarrow X$ the inclusion of the stable locus and by $\pi: X^{s} \rightarrow Y$ the projection to the quotient. An equivariant sheaf $\mathcal{E}$ descends to $Y$ if there exists a sheaf $\check{\mathcal{E}}$ on $Y$ such that $\pi^{*} \check{\mathcal{E}}$ is equivariantly isomorphic to $\iota^{*} \mathcal{E}$. In [19], Nevins gave a general criterion for the descent of a sheaf through a good quotient. In Section 3.2, we give a combinatorial criterion for the descent of equivariant reflexive sheaves under generic toric GIT quotients. We then build the functors $\mathfrak{P}_{\mathbf{i}}$, by extending equivariant reflexive sheaves pulled-back from $Y$ to $X^{s}$ across the unstable locus (see [11] for similar constructions). The elements in the images of these functors are described geometrically, and correspond precisely to the reflexive sheaves that descend to $Y$ and for which the slopes on $X$ and $Y$ will be comparable. The second part of the proof of Theorem 1.1, given in Section 4. gives a relation between slopes on $(X, L)$ and slopes on $(Y, \alpha)$. A combinatorial 
formula describes these slopes [4,9]. In this formula, there are contributions from the sheaves and from the polarizations. The functors $\mathfrak{P}_{\mathrm{i}}$ are precisely constructed so that the sheaf contributions can be compared on $X$ and $Y$. As for the polarization terms, they are related to the volumes of the facets of the associated polytopes. To be able to compare them through the quotient, we use a classical result of Minkowski stating precisely when the volumes of the facets of a polytope can be prescribed.

Remark 1.6. It might be worth noting that part of our results are not inherent to the toric world and should admit more general formulations. Given a polarized variety $(X, L)$ with a linearized action of a reductive group $G$ and with GIT quotient $Y$, one should be able to construct functors $\mathfrak{P}_{\mathbf{i}}$ from the category of reflexive sheaves on $Y$ to the category of $G$-equivariant reflexive sheaves on $X$ as we do in Section 3.3 However, being able to determine a class $\alpha \in N^{1}(Y)_{\mathbb{R}}$ such that the slopes of the appropriate sheaves on $X$ and $Y$ can be compared seems to be a much more delicate problem in such generality. This should be possible in some specific situations though, and we hope to obtain generalizations of Theorem 1.1 for varieties with large symmetry groups, such as $T$-varieties of low complexity or spherical varieties.

The organization of the paper is as follows. In Section 2 we gather standard facts about toric varieties and equivariant reflexive sheaves that will be used in the paper. In particular, we recall in Section 2.1 the classical correspondence between polytopes and polarized toric varieties, and describe its generalization to real ample divisors. In Section 2.2, we recall Klyachko's description of the category of equivariant reflexive sheaves on toric varieties. Along the way, we give a new and shorter proof for the combinatorial formula for the first Chern class of these objects, extending several earlier results to normal toric varieties (compare with [4,9,14]). Section 3 deals with the descent of reflexive sheaves under toric GIT. We start by recalling the necessary material of toric GIT in Section 3.1, then prove a descent criterion in Section 3.2, and last construct the pullback functors in Section 3.3. With this material at hand, we can prove Theorem 1.1 in Section 4. Together with Section 3, they form the core of the paper. We first introduce the notions of slope stability in Section 4.1, and then recall a classical theorem of Minkowski in Section 4.2. to conclude with the proof of our main theorem. Finally, in Section 5 we study compatible actions and give applications of our result, proving Proposition 1.2 and Corollary 1.4.

Acknowledgments. We would like to thank Alberto Della Vedova, Henri Guenancia, Johannes Huisman, Éveline Legendre, Yann Rollin and Hendrik Süss for stimulating discussions. We are also grateful to the referees for useful comments that helped in improving the paper. The first named author would like to acknowledge the financial support of the CNRS and CAPES-COFECUB that made possible his visit to LMBA.

\section{EquiVARIANT REFLEXIVE SHEAVES ON TORIC VARIETIES}

Throughout this paper, we consider toric varieties over the complex numbers. We recall the description of polarized toric varieties in terms of polytopes and the characterization of equivariant reflexive sheaves on toric varieties in terms of families of filtrations. 
2.1. Polarized toric varieties and polytopes. We refer to [2, Chapters 2, 3,6] and 22 for this section. Let $N$ be a rank $n$ lattice, $M:=\operatorname{Hom}_{\mathbb{Z}}(N, \mathbb{Z})$ be its dual with pairing $\langle\cdot, \cdot\rangle$. Then $N$ is the lattice of 1-parameter subgroups of a $n$-dimensional complex torus

$$
T:=N \otimes_{\mathbb{Z}} \mathbb{C}^{*}=\operatorname{Hom}_{\mathbb{Z}}\left(M, \mathbb{C}^{*}\right) .
$$

We set $N_{\mathbb{F}}=N \otimes_{\mathbb{Z}} \mathbb{F}$ and $M_{\mathbb{F}}=M \otimes_{\mathbb{Z}} \mathbb{F}$ for $\mathbb{F}=\mathbb{Q}$ or $\mathbb{R}$.

Let $X=X(\Sigma)$ be an $n$-dimensional projective toric variety associated to a fan $\Sigma$, so that in particular $X$ is normal. Denote $\Sigma=\left\{\sigma_{i}: i \in I\right\}$, with $\sigma_{i}$ a strongly convex rational polyhedral cone in $N_{\mathbb{R}}$ for all $i \in I$. Denote also by $\Sigma(k)$ the set of $k$-dimensional cones in $\Sigma$. The variety $X$ is obtained by gluing affine charts $\left(U_{\sigma}\right)_{\sigma \in \Sigma}$, with

and $\mathbb{C}\left[S_{\sigma}\right]$ is the semi-group algebra of

$$
U_{\sigma}=\operatorname{Spec}\left(\mathbb{C}\left[S_{\sigma}\right]\right)
$$

$$
S_{\sigma}=\sigma^{\vee} \cap M=\{m \in M:\langle m, n\rangle \geq 0 \text { for all } n \in \sigma\} .
$$

There exists a bijective correspondence between cones $\sigma \in \Sigma$ and $T$-orbits $O(\sigma)$ in $X$. This satisfies, for $\sigma \in \Sigma, \operatorname{dim} O(\sigma)=n-\operatorname{dim}(\sigma)$. In particular, to any $\rho \in \Sigma(1)$, there corresponds a irreducible $T$-invariant Weil divisor $D_{\rho}$ given by

$$
D_{\rho}=\overline{O(\rho)}
$$

where the closure is in both classical and Zariski topologies.

As $X$ is associated to the fan $\Sigma$, there is a bijective correspondence between $T$-invariant ample divisors on $X$ and lattice polytopes $P \subset M_{\mathbb{R}}$ whose normal fan $\Sigma_{P}$ is equal to $\Sigma$ (see [2, Theorem 6.2.1]). Let $D$ be a $T$-invariant Cartier divisor on $X$. Recall that it is equal to a linear combination of the form

$$
D=\sum_{\rho \in \Sigma(1)} a_{\rho} D_{\rho} .
$$

For each $\rho \in \Sigma(1)$ we denote by $u_{\rho} \in N$ the minimal generator of $\rho \cap N$. Assuming that $D$ is ample, we consider the associated polytope $P=P_{D} \subset M_{\mathbb{R}}$ :

$$
P=\left\{m \in M_{\mathbb{R}}:\left\langle m, u_{\rho}\right\rangle \geq-a_{\rho} \text { for all } \rho \in \Sigma(1)\right\} .
$$

Note that if $D^{\prime}$ is equivariant and linearly equivalent to $D$ then $P_{D^{\prime}}$ is given by translation of $P_{D}$ in $M$ by some lattice element $m \in M$. In the same way, a lattice translation of the polytope $P_{D}$ corresponds to a different linearization of the action of $T$ on the line bundle $\mathcal{O}(D)$ (see Section 3.1).

As $D$ is ample, and $P$ has normal fan equal to $\Sigma$, we have a correspondence between cones in $\Sigma$ and faces of $P$. For a face $Q$ in $P$, we denote by $\sigma_{Q} \in \Sigma$ (resp. by $O(Q))$ the associated cone (resp. the associated orbit). In particular, rays in $\Sigma$ corresponds to facets of $P$. For each $\rho \in \Sigma(1)$ the associated facet is

$$
F=P \cap\left\{m \in M_{\mathbb{R}}:\left\langle m, u_{\rho}\right\rangle=-a_{\rho}\right\} .
$$

We will denote $u_{\rho}$ by $u_{F}$ and $a_{\rho}$ by $a_{F}$. We can also write

$$
D=\sum_{F \prec P} a_{F} D_{F},
$$

where the sum is over all facets of $P$ and $D_{F}:=D_{\rho_{F}}$. We will denote faces of $P$ of higher codimension by the letter $Q$ and vertices by the letter $v$. We use the relation $Q_{1} \preccurlyeq Q_{2}$ to signify that $Q_{1}$ and $Q_{2}$ are faces of $P$, possibly equal to $P$ itself, and $Q_{1} \subseteq Q_{2}$. 
The correspondence between polarizations on $X$ and lattice polytopes with normal fan $\Sigma$ modulo lattice translations extends to real ample classes. We recall the definition of real ample divisors on a normal complex algebraic variety $X$ (see [15]). Let $\operatorname{Div}(X)$ denote the group of integral Cartier divisors on $X$. The Néron-Severi group is given by $N^{1}(X)=\operatorname{Div}(X) / \sim_{n u m}$ and we set $N^{1}(X)_{\mathbb{R}}=N^{1}(X) \otimes_{\mathbb{Z}} \mathbb{R}$. Denote by $\operatorname{WDiv}(X)$ the set of Weil divisors on $X$ and $\operatorname{WDiv}_{\mathbb{R}}(X)$ the vector space of real Weil divisors.

Definition 2.1. A class $\alpha \in N^{1}(X)_{\mathbb{R}}$ is ample if it is represented by a positive real linear combination of ample Cartier divisors.

We then have the following:

Proposition 2.2. Let $X$ be a projective toric variety given by a fan $\Sigma$. Then there is a bijective correspondence between real ample classes on $X$ and real polytopes of the form

$$
P=\left\{m \in M_{\mathbb{R}}:\left\langle m, u_{\rho}\right\rangle \geq-a_{\rho}, \text { for all } \rho \in \Sigma(1)\right\}
$$

for which the normal fan $\Sigma_{P}=\Sigma$, modulo real translations within $M_{\mathbb{R}}$.

Proof. This statement between integral classes and lattice polytopes is standard (note that for projective toric varieties coming from fans, the real Picard group and the real Néron-Severi group coincide, see [2, Proposition 6.3.15]). The rational case follows by clearing denominators and scaling polytopes. We now prove the real case. Set $\operatorname{Pic}(X)$ and $\operatorname{Cl}(X)$ the Picard and class groups of $X$. From the exact sequence (see 2, Theorem 4.1.3]):

$$
0 \longrightarrow M \longrightarrow \bigoplus_{\rho \in \Sigma(1)} \mathbb{Z} \cdot D_{\rho} \stackrel{\pi}{\longrightarrow} \mathrm{Cl}(X) \longrightarrow 0
$$

we deduce the sequences of vector spaces, for $\mathbb{K}=\mathbb{Q}$ or $\mathbb{R}$ :

$$
0 \longrightarrow M_{\mathbb{K}} \longrightarrow W_{\mathbb{K}} \longrightarrow \operatorname{Pic}(X)_{\mathbb{K}} \longrightarrow 0
$$

where we set $W=\pi^{-1}(\operatorname{Pic}(X)), W_{\mathbb{K}}=W \otimes_{\mathbb{Z}} \mathbb{K}$ and $\operatorname{Pic}(X)_{\mathbb{K}}=\operatorname{Pic}(X) \otimes_{\mathbb{Z}} \mathbb{K}$.

Let $\alpha \in \operatorname{Pic}(X)_{\mathbb{R}}=N^{1}(X)_{\mathbb{R}}$ be an ample class. We can represent $\alpha$ by

$$
D=\sum_{\rho \in \Sigma(1)} a_{\rho} D_{\rho} \in W_{\mathbb{R}} .
$$

Define the set

$$
P_{\alpha}=\left\{m \in M_{\mathbb{R}}: \forall \rho \in \Sigma(1),\left\langle m, u_{\rho}\right\rangle \geq-a_{\rho}\right\} .
$$

First observe that $P_{\alpha}$ is a polytope, rather than a polyhedron, since the fan $\Sigma$ is complete. By definition of ample real divisors, $D=\sum_{i=1}^{N} \alpha_{i} D_{i}$ for $D_{i}$ ample Cartier divisors and $\alpha_{i}$ positive real numbers. By the openness of the ampleness condition, we can take positive rational numbers $\beta_{i} \in \mathbb{Q}$ as close as we like to the $\alpha_{i} \in \mathbb{R}$ in such a way that

$$
D_{\beta}=\sum_{i=1}^{N} \beta_{i} D_{i}=\sum_{\rho \in \Sigma(1)} b_{\rho} D_{\rho}
$$

is an ample $\mathbb{Q}$-divisor, and hence the polytope

$$
P_{\beta}=\left\{m \in M_{\mathbb{R}}: \forall \rho \in \Sigma(1),\left\langle m, u_{\rho}\right\rangle \geq-b_{\rho}\right\}
$$


has normal cone $\Sigma_{P_{\beta}}=\Sigma$. Furthermore, decomposing the divisors $D_{i}$ in the basis $\left\{D_{\rho}: \rho \in \Sigma(1)\right\}$, we see that the values $\left(b_{\rho}\right)$ vary continuously with respect to $\left(\beta_{i}\right)$, so can be made sufficiently close to the $\left(a_{\rho}\right)$ to guarantee that $\Sigma_{P_{\alpha}}=\Sigma_{P_{\beta}}=\Sigma$. Note that as in the integral case, translations of $P_{\alpha}$ by elements of $M_{\mathbb{R}}$ correspond to different choices of representative of the class $\alpha$ in $W_{\mathbb{R}}$.

For the converse statement, we consider the polytope in $M_{\mathbb{R}}$

$$
P=\left\{m \in M_{\mathbb{R}}: \forall \rho \in \Sigma(1),\left\langle m, u_{\rho}\right\rangle \geq-a_{\rho}\right\}
$$

for $a_{\rho} \in \mathbb{R}$, supposing that the normal fan of $P$ determined by the vectors $u_{\rho}$ is the fan of $X$. Then, the polytope $P$ determines the real Weil divisor $D_{P}=$ $\sum_{\rho} a_{\rho} D_{\rho} \in \operatorname{WDiv}_{\mathbb{R}}(X)$. We show that $D_{P}$ lies in the space of real Cartier divisors, and is moreover ample. This is proven by induction on the number of $a_{\rho}$ 's that are irrational. We list the rays in $\Sigma$ by $\rho_{1}, \ldots, \rho_{d}$ for $d=\# \Sigma(1)$. As noted above, the case where all $a_{\rho}$ 's are rational is well-known. Suppose that, for fixed $k \geq 1$, $D=\sum_{i=1}^{d} a_{\rho_{i}} D_{\rho_{i}}$ defines a real ample class whenever its normal fan $\Sigma_{P}=\Sigma$ and $a_{\rho_{i}} \in \mathbb{Q}$ for all $i \geq k$. If $a_{\rho_{i}} \in \mathbb{Q}$ for $i \geq k+1$ then let $r_{1}, r_{2}$ be rational numbers for which $r_{1}<a_{\rho_{k}}<r_{2}$ sufficiently close to $a_{\rho_{k}}$ that for any $s \in\left[r_{1}, r_{2}\right]$ the polytope defined by the inequalities

$$
\begin{aligned}
& \left\langle m, u_{\rho_{i}}\right\rangle \geq-a_{\rho_{i}}, \quad \text { for } i \neq k, \\
& \left\langle m, u_{\rho_{k}}\right\rangle \geq-s
\end{aligned}
$$

defines the same normal fan $\Sigma$. Then, for some $t \in[0,1]$, we have $a_{\rho_{k}}=t r_{1}+(1-t) r_{2}$ and

$$
D=\sum_{i=1}^{d} a_{\rho_{i}} D_{\rho_{i}}=t\left(r_{1} D_{\rho_{k}}+\sum_{i \neq k} a_{\rho_{i}} D_{\rho_{i}}\right)+(1-t)\left(r_{2} D_{\rho_{k}}+\sum_{i \neq k} a_{\rho_{i}} D_{\rho_{i}}\right) .
$$

By the induction hypothesis, each of the two real divisors on the right hand side of the above equality is ample. By the convexity of the set of real ample classes, $D$ is ample.

Remark 2.3. We note that in the smooth case, a similar result can be given via symplectic geometry by using the correspondence between compact symplectic toric manifolds and Delzant polytopes up to translations.

2.2. Equivariant reflexive sheaves. We refer to [12, 14, 20] for this section. We consider a projective toric variety $X$ together with a polytope $P \subset M_{\mathbb{R}}$ associated to an ample class on $X$. We are interested in a combinatorial description of equivariant sheaves on $X$.

Remark 2.4. From now on, by equivariant sheaf on $X$ we will mean equivariant with respect to the torus $T$ of $X$. When later we consider equivariant sheaves on $Y$ a GIT quotient of $X$, we will mean equivariant with respect to the torus of $Y$.

Recall that a reflexive sheaf on $X$ is a coherent sheaf $\mathcal{E}$ that is canonically isomorphic to its double dual $\mathcal{E}^{\vee \vee}$. Klyachko gave a description of equivariant reflexive sheaves in terms of combinatorial data:

Definition 2.5. A family of filtrations $\mathbb{E}$ is the data of a finite dimensional vector space $E$ and for each facet $F$ of $P$, an increasing filtration $\left(E^{F}(i)\right)_{i \in \mathbb{Z}}$ of $E$ such that $E^{F}(i)=\{0\}$ for $i \ll 0$ and $E^{F}(i)=E$ for some $i$. We will denote by $i_{F}$ the smallest $i \in \mathbb{Z}$ such that $E^{F}(i) \neq 0$. 
Remark 2.6. Families of filtrations in [12] or 20] are labeled by the set of rays $\rho \in \Sigma(1)$. As $P$ is associated to an ample class, there is a one-to-one correspondence between the facets of $P$ and the rays of the fan of $X$, and so we recover the usual definition. Note also that we use increasing filtrations of $E$, as in [20], rather than decreasing as in 12 .

To a family of filtrations $\mathbb{E}:=\left\{\left(E^{F}(i)\right) \subseteq E: F \prec P, i \in \mathbb{Z}\right\}$ we can assign an equivariant reflexive sheaf $\mathcal{E}:=\mathfrak{K}(\mathbb{E})$ defined by

$$
\Gamma\left(U_{\sigma_{Q}}, \mathcal{E}\right):=\bigoplus_{m \in M} \bigcap_{Q \preccurlyeq F} E^{F}\left(\left\langle m, u_{F}\right\rangle\right) \otimes \chi^{m}
$$

for all proper faces $Q \prec P$, while $\Gamma\left(U_{\sigma_{P}}, \mathcal{E}\right)=E \otimes \mathbb{C}[M]$.

Remark 2.7. The conditions for a family of filtrations to define a locally-free sheaf are determined in [12].

The morphisms of families of filtrations are defined by:

Definition 2.8. A morphism between two families of filtrations $\mathbb{E}_{1}=\left\{\left(E_{1}^{F}(i)\right) \subseteq E_{1}\right.$ : $F \prec P, i \in \mathbb{Z}\}$ and $\mathbb{E}_{2}=\left\{\left(E_{2}^{F}(i)\right) \subset E_{2}: F \prec P, i \in \mathbb{Z}\right\}$ is a linear map $\phi: E_{1} \rightarrow E_{2}$ preserving the filtrations, that is such that for all $F$ and all $i, \phi\left(E_{1}^{F}(i)\right) \subset E_{2}^{F}(i)$.

Such a morphism between families of filtrations induces an equivariant morphism between the associated reflexive sheaves.

Remark 2.9. In this paper, by a morphism between equivariant reflexive sheaves $\mathcal{E}_{1}=\mathfrak{K}\left(\mathbb{E}_{1}\right)$ and $\mathcal{E}_{2}=\mathfrak{K}\left(\mathbb{E}_{2}\right)$, we mean an equivariant morphism of degree zero: the space $\operatorname{Hom}\left(\mathcal{E}_{1}, \mathcal{E}_{2}\right)$ of all sheaves homomorphisms is naturally graded over $M$ :

$$
\operatorname{Hom}\left(\mathcal{E}_{1}, \mathcal{E}_{2}\right) \subset \operatorname{Hom}\left(E_{1}, E_{2}\right) \otimes \mathbb{C}[M]
$$

and the morphisms that we will consider are those lying in the $\operatorname{Hom}\left(E_{1}, E_{2}\right) \otimes$ $\chi^{0}$-component of the grading. In particular, this excludes injective maps like $\mathcal{O}_{\mathbb{P}^{1}}(-1) \rightarrow \mathcal{O}_{\mathbb{P}^{1}}$

For a toric variety $Z$, and an ample polytope $P_{Z}$, we denote by:

i) $\mathfrak{R e f} \mathfrak{f}^{T}(Z)$ the category of equivariant reflexive sheaves on $Z$,

ii) $\mathfrak{F i l t}\left(P_{Z}\right)$ the category of families of filtrations associated to $P_{Z}$.

From Klyachko and Perling [12,20, we obtain the following:

Theorem 2.10. [12,20, The functor $\mathfrak{K}$ induces an equivalence of categories between the category $\mathfrak{F i l t}(P)$ and the category $\mathfrak{R e f}^{T}(X)$.

As the category of filtrations on a given finite dimensional vector space is abelian, we have:

Corollary 2.11. The category $\mathfrak{R e f}^{T}(X)$ of equivariant reflexive sheaves on $X$ is an abelian category.

We will need the combinatorial characterizations of equivariant reflexive saturated subsheaves and of equivariant rank 1 reflexive sheaves. Let $\mathcal{E}=\mathfrak{K}(\mathbb{E})$ be an equivariant reflexive sheaf on $X$, given by a family of filtrations $\mathbb{E}=\left\{\left(E^{F}(i)\right) \subset\right.$ $E: F \prec P, i \in \mathbb{Z}\}$. For any vector subspace $W \subset E$, define a family of filtrations $\mathbb{E} \cap W$ by

$$
\mathbb{E} \cap W=\left\{\left(W \cap E^{F}(i)\right) \subset W \cap E: F \prec P, i \in \mathbb{Z}\right\} .
$$

Then, the sheaf $\mathcal{E}_{W}:=\mathfrak{K}(\mathbb{E} \cap W)$ is an equivariant saturated reflexive subsheaf of $\mathcal{E}$. Any equivariant reflexive saturated subsheaf of $\mathcal{E}$ arises that way: 
Proposition 2.12. ( 9, Rem. 2.4.]) Let $\mathcal{E}=\mathfrak{K}(\mathbb{E})$ be an equivariant reflexive sheaf on $X$. Let $\mathcal{F} \subset \mathcal{E}$ be an equivariant saturated reflexive subsheaf of $\mathcal{E}$. Then, there is a unique vector subspace $W \subset E$ such that $\mathcal{F}=\mathfrak{K}(\mathbb{E} \cap W)$.

As for rank 1 reflexive sheaves, from the definition we obtain:

Proposition 2.13. Let $\mathcal{O}(-D)$ be the rank 1 reflexive sheaf associated to the invariant Weil divisor $D=\sum_{F \prec P} a_{F} D_{F}$. Then, $\mathcal{O}(-D)=\mathfrak{K}\left(\mathbb{E}_{D}\right)$, where the family of filtrations $\mathbb{E}_{D}=\left\{\left(E^{F}(i)\right) \subset \mathbb{C}, F \prec P, i \in \mathbb{Z}\right\}$ satisfies

$$
E^{F}(i)=\left\{\begin{array}{lll}
0 & \text { if } \quad i<a_{F} \\
\mathbb{C} & \text { if } \quad i \geq a_{F}
\end{array}\right.
$$

We will also need the determinant and first Chern class of reflexive sheaves.

Remark 2.14. Let $A_{k}(X)$ be the $k$-th Chow group of $X$. This is the quotient of the free abelian group on $k$-dimensional subvarieties by rational equivalence. The first Chern class is the map $c_{1}: \operatorname{Pic}(X) \rightarrow A_{n-1}(X)$ induced by the inclusion of Cartier divisors in Weil divisors. This defines a morphism $A_{k}(X) \rightarrow A_{k-1}(X)$ for each $k$ as follows. For $\mathcal{L}$ a line bundle and $V$ a $k$-dimensional subvariety on $X,\left.\mathcal{L}\right|_{V}$ defines a Cartier divisor on $V$, hence a $(k-1)$-cycle on $X$.

As $X$ is normal, this definition extends to rank one reflexive sheaves since every rank-one reflexive sheaf is of the form $\mathcal{E}=\mathcal{O}_{X}\left(D_{\mathcal{E}}\right)$ for some Weil divisor $D_{\mathcal{E}}$, and hence $c_{1}(\mathcal{E}):=\left[D_{\mathcal{E}}\right] \in A_{n-1}(X)$. If $H$ is an ample line bundle on $X$, the degree of $\mathcal{E}$ is then given by

$$
\operatorname{deg}_{H}(\mathcal{E})=c_{1}(\mathcal{E}) \cdot H^{n-1} \in A_{0}(X) \cong \mathbb{Z} .
$$

Recall the following:

Definition 2.15. If $\mathcal{E}$ is a torsion-free coherent sheaf on $X$, one defines the determinant of $\mathcal{E}$ to be the rank-one reflexive sheaf $\operatorname{det}(\mathcal{E})=\left(\Lambda^{\mathrm{rk}(\mathcal{E})} \mathcal{E}\right)^{\vee \vee}$. Then, the first Chern class of $\mathcal{E}$ is $c_{1}(\mathcal{E}):=c_{1}(\operatorname{det} \mathcal{E})$.

We prove now that the previously known formula for the first Chern class for equivariant reflexive sheaves holds in our case, as a linear combination of Weil divisors. This extends the previous case on smooth varieties, given by Kool, to normal varieties. To produce a necessary combinatorial formula, we first need to introduce some notation:

Definition 2.16. Let $\mathcal{E}=\mathfrak{K}(\mathbb{E})$, with $\mathbb{E}=\left\{\left(E^{F}(i)\right) \subset E: F \prec P, i \in \mathbb{Z}\right\}$. We set, for all $F \prec P$ and all $i \in \mathbb{Z}$ :

$$
e^{F}(i)=\operatorname{dim}\left(E^{F}(i)\right)-\operatorname{dim}\left(E^{F}(i-1)\right) .
$$

We will refer to the integers $\left(e^{F}(i)\right)_{F \prec P, i \in \mathbb{Z}}$ as the dimension jumps of $\mathbb{E}$ or $\mathcal{E}$.

Then we have:

Proposition 2.17. Let $\mathcal{E}=\mathfrak{K}(\mathbb{E})$ be a rank $r$ equivariant reflexive sheaf on $X$, given by a family of filtrations $\mathbb{E}=\left\{\left(E^{F}(i)\right) \subset E: F \prec P, i \in \mathbb{Z}\right\}$. We define a family of filtrations $\operatorname{det}(\mathbb{E})=\left\{\left(E_{\operatorname{det}}^{F}(i)\right) \subset \Lambda^{r} E: F \prec P, i \in \mathbb{Z}\right\}$ by setting, for all $F \prec P$,

$$
E_{\operatorname{det}}^{F}(i)=\left\{\begin{array}{ccc}
0 & \text { if } \quad i<i_{F}(\operatorname{det} \mathcal{E}) \\
\Lambda^{r} E & \text { if } \quad i \geq i_{F}(\operatorname{det} \mathcal{E})
\end{array}\right.
$$


where for all $F \prec P$,

$$
i_{F}(\operatorname{det} \mathcal{E})=\sum_{i \in \mathbb{Z}} i e^{F}(i)
$$

Then $\operatorname{det}(\mathcal{E})=\mathfrak{K}(\operatorname{det}(\mathbb{E}))$.

Proof. Note first that because $\mathfrak{R e f}{ }^{T}(X)$ is abelian, $\Lambda^{r} \mathcal{E}$ is reflexive and $\operatorname{det}(\mathcal{E})=$ $\Lambda^{r} \mathcal{E}$. Then, the family of filtrations $\left\{\left(\Lambda^{r} E^{F}(i)\right): F \prec P, i \in \mathbb{Z}\right\}$ for $\Lambda^{r} \mathcal{E}$ satisfies:

$$
\Lambda^{r} E^{F}(i)=\sum_{i_{1}, \ldots, i_{r}: \sum i_{j}=i} E^{F}\left(i_{1}\right) \wedge \ldots \wedge E^{F}\left(i_{r}\right) .
$$

Now, $\Lambda^{r} E$ is one dimensional, so

$$
\Lambda^{r} E^{F}(i)=\left\{\begin{array}{ccc}
0 & \text { if } & i<j_{F} \\
\Lambda^{r} E & \text { if } & i \geq j_{F}
\end{array}\right.
$$

where $j_{F}$ is the smallest integer $l \in \mathbb{Z}$ such that there is a partition $i_{1}, \ldots, i_{r}$ of $l$ with $E^{F}\left(i_{1}\right) \wedge \ldots \wedge E^{F}\left(i_{r}\right) \neq\{0\}$. From the fact that $\left(E^{F}(i)\right)$ forms a filtration of vector spaces, we deduce that $j_{F}$ must be the sum of the integers $i$ such that the dimension of $E^{F}(i)$ changes, counted with multiplicity. Hence $j_{F}=\sum_{i \in \mathbb{Z}} i e^{F}(i)$, which ends the proof.

Corollary 2.18. Let $\mathcal{E}=\mathfrak{K}(\mathbb{E})$ be an equivariant reflexive sheaf on $X$, given by a family of filtrations $\mathbb{E}=\left\{\left(E^{F}(i)\right) \subset E: F \prec P, i \in \mathbb{Z}\right\}$. The first Chern class of $\mathcal{E}$ is the class of the Weil divisor:

$$
c_{1}(\mathcal{E})=-\sum_{F \prec P} i_{F}(\operatorname{det} \mathcal{E}) D_{F} .
$$

where for all $F \prec P$,

$$
i_{F}(\operatorname{det} \mathcal{E})=\sum_{i \in \mathbb{Z}} i e^{F}(i)
$$

Remark 2.19. In this paper, we restrict ourselves to reflexive sheaves. We expect that most of the results extend to equivariant torsion-free coherent sheaves, described in terms of families of multifiltrations [14, 20. Since the applications we have in mind concern stable vector bundles, it is enough to consider the category of reflexive sheaves, where the results and proofs are simpler to express.

\section{Descent of Equivariant Sheaves under toric GIT}

In this section we study the descent of equivariant reflexive sheaves under toric GIT quotients. We denote by $X$ a projective toric variety, polarized by an ample equivariant line bundle $L$. We retain the notation of the previous section.

3.1. Toric GIT. We refer to [17,22 for this section. We are interested in GIT quotients of $(X, L)$ by subtorus actions. Let $N_{0}$ be a sublattice of $N$ of rank $g$. We will assume that $N_{0}$ is saturated, that is $N_{0}=\left(N_{0} \otimes_{\mathbb{Z}} \mathbb{R}\right) \cap N$. The sublattice $N_{0}$ spans a $g$-dimensional subtorus $G=N_{0} \otimes_{\mathbb{Z}} \mathbb{C}^{*}$ of $T$. We fix a linearization $\gamma$ of $T$ on $L$ and we will consider the GIT quotient with respect to the induced linearization of $G$. From Section 2.1] $(X, L)$ defines a family of lattice polytopes $\left\{P_{D}: \mathcal{O}(D) \sim L\right\}$, all equal up to translations by lattice elements. Then, the linearization $\gamma$ determines a unique polytope $P$ in this family such that

$$
H^{0}(X, L)=\bigoplus_{m \in P \cap M} \mathbb{C} \cdot \chi^{m}
$$


is the weight decomposition of the $T$-action on the space of global sections induced by $\gamma$ (see [2, Proposition 4.3.3]). We have the following:

$$
0 \rightarrow N_{0} \rightarrow N \rightarrow N / N_{0} \rightarrow 0
$$

and the associated dual sequence:

$$
0 \rightarrow N_{0}^{\perp} \rightarrow M \rightarrow M / N_{0}^{\perp} \rightarrow 0 .
$$

Set $U=N_{0}^{\perp} \otimes_{\mathbb{Z}} \mathbb{R} \subset M_{\mathbb{R}}$. Then from [22, Proposition 3.2]:

Proposition 3.1. The GIT quotient of $(X, L)$ by $G$ is the polarized toric variety $(Y, \check{L})$ described by the polytope $P_{Y}=U \cap P$. Moreover, its lattice is $N_{Y}=N / N_{0}$ with dual $N_{0}^{\perp}$.

Remark 3.2. Note that the vertices of the polytope $P \cap U$ are not necessarily lattice points, so this polytope only induces a rational polarization $\check{L}$ on the variety $Y=X / / G$.

Remark 3.3. Up to composition by a finite morphism, we can always reduce to the case $N_{0}$ saturated. In this paper, we will restrict ourselves to this simpler situation.

We can also describe the stable and semistable points :

Proposition 3.4 ( [22], Lemma 3.3). The semistable and stable loci $X^{s s}$ and $X^{s}$ under the $G$ action on $(X, L)$ are each unions of $T$-orbits. More precisely, given a face $Q \preccurlyeq P$, the orbit $O(Q)$ is:

- semistable iff $Q \cap U \neq \varnothing$,

- stable iff $Q \cap U \neq \varnothing$ and the interior of $Q$ meets $U$ transversally.

From this proposition, the set of faces of the polytope of $X^{s s} / / G$ is

$$
\{U \cap Q: Q \preccurlyeq P\} \text {. }
$$

We will denote by $P^{s}$ (resp. $P^{s s}$ and $P^{u s}$ ) the set of faces corresponding to stable orbits (resp. semistable orbits and unstable orbits) of $X$. For technical reasons, we will need the following assumption on the action:

Definition 3.5. A pair of a subtorus $G \subset T$ and linearization $\gamma: T \rightarrow \operatorname{Aut}(L)$ will be called generic if the stable and semi-stable loci of the $G$ action on $(X, L)$ coincide and are not empty.

The nice fact about generic actions is the following:

Lemma 3.6. Assume that $(G, \gamma)$ is generic. Then:

i) The points of $X^{s}$ have finite stabilizers under the $G$-action.

ii) There is a 1:1-correspondence between facets of $P_{Y}$ and facets in $P^{s}$.

Proof. The first point follows by definition of stable points. For the second point, any facet of $U \cap P$ must be of the form $U \cap Q$ for some face $Q$ of $P$. By assumption, $Q$ meets $U$ transversally. Moreover, $\operatorname{dim}(U \cap Q)=\operatorname{dim}(Y)-1=n-1-g$, where $g$ is the rank of the sub-lattice $N_{0} \subseteq N$ and equal to the dimension of the subgroup $G$. This forces the dimension of $Q$ to be $n-1$, hence the result.

From now on, unless explicitly stated, we will assume that $(G, \gamma)$ is generic. We end this section with some definitions and lemmas relating the primitive normals to the facets of $P$ to those of $P_{Y}$. We consider the lattice projection, $\pi: N \rightarrow N_{Y}=$ $N / N_{0}$. 
Definition 3.7. For each facet $F \cap U$ of $P_{Y}$, set $\check{u}_{F}$ to be the primitive element in $N_{Y}$ defining $F \cap U$ as an inner pointing normal.

Lemma 3.8. There exists a unique positive integer $b_{F}$ such that

$$
\check{u}_{F}=\frac{1}{b_{F}} \pi\left(u_{F}\right) .
$$

Proof. Both elements lies on the ray $\rho_{F \cap U}$, and $\check{u}_{F}$ generates this ray.

Let $F$ be facet in $P^{s}$. As $u_{F}$ is primitive, we can complete it into a basis $\mathcal{B}_{F}:=\left\{u_{F}, u_{i}: i=2 \ldots n\right\}$ of $N$. We denote by $\mathcal{B}_{F}^{*}=\left\{m_{F}, m_{i}: i=2 \ldots n\right\}$ the dual basis of $M$. In particular, note that $\left\{m_{i}: i=2 \ldots n\right\}$ is a basis for $u_{F}^{\perp} \cap M$. Similarly, we set $\check{\mathcal{B}}_{F}:=\left\{\check{u}_{F}, \check{u}_{i}: i=2 \ldots n-g\right\}$ a basis for $N_{Y}$ with dual basis $\check{\mathcal{B}}_{F}^{*}:=\left\{\check{m}_{F}, \check{m}_{i}: i=2 \ldots n-g\right\}$ and again $\left\{\check{m}_{i}: i=2 \ldots n-g\right\}$ is a basis for $\check{u} \frac{\perp}{F} \cap N_{Y}$. From Lemma 3.8 , we deduce:

Lemma 3.9. The element $\check{m}_{F} \in N_{0}^{\perp} \subset M$ can be uniquely written as

$$
\check{m}_{F}=b_{F} m_{F}+m_{F}^{\perp}
$$

with $m_{F}^{\perp} \in u_{F}^{\perp}$.

We conclude this section with the following observation:

Remark 3.10. If $X$ is smooth, it is easy to show in a local chart $U_{F}=\operatorname{Spec}\left(\mathbb{C}\left[\sigma_{F}^{\vee} \cap\right.\right.$ $M]$ ) that $b_{F}$ is the order of the stabilizer of the orbit $\mathcal{O}(F)$ under the $G$-action.

3.2. Descent criteria for equivariant reflexive sheaves. We retain the notation from the previous section. We assume as before that $(X, L)$ is a polarized toric variety of dimension $n$ and that $(G, \gamma)$ is generic. We consider $(Y, \check{L})$ the GIT quotient associated to the $G$ action on $(X, L)$. Following [22] and the discussion in the previous section, we recall that $Y$ is a simplicial toric variety, with torus given by $T_{Y}=T_{X} / G$, and $\check{L}$ is the invariant ample line bundle on $Y$ corresponding to the polytope $P_{Y}=P \cap U$. We wish to compare equivariant reflexive sheaves on $X$ and equivariant reflexive sheaves on $Y$. We denote by $\iota: X^{s} \rightarrow X$ the inclusion and by $\pi: X^{s} \rightarrow Y$ the projection. We then introduce:

Definition 3.11. We say that a $G$-equivariant coherent sheaf $\mathcal{E}$ on $X$ descends to $Y$ if there is a coherent sheaf $\mathcal{F}$ on $Y$ such that $\pi^{*} \mathcal{F}$ is $G$-equivariantly isomorphic to $\iota^{*} \mathcal{E}$.

Let $\mathcal{E}$ be a $G$-equivariant coherent sheaf on $X$. For simplicity we will denote by $\mathcal{E}^{s}$ the restriction $\iota^{*} \mathcal{E}$. As explained for example in [19, $\mathcal{E}$ descends to $Y$ if and only if

$$
\mathcal{E}^{s} \simeq \pi^{*} \pi_{*}^{G} \mathcal{E}^{s}
$$

where $\pi_{*}^{G}$ is the invariant pushforward.

Remark 3.12. Note that the functors $\iota^{*}, \pi^{*}$ and $\pi_{*}^{G}$ preserve the torus equivariantness and reflexivity properties of coherent sheaves (for $\pi^{*}$, it follows from flatness of $\pi)$.

We will give a description of the functors $\iota^{*}, \pi^{*}$ and $\pi_{*}^{G}$ for torus-equivariant reflexive sheaves in terms of families of filtrations. Making use of the equivalence of categories $\mathfrak{K}$, by abuse of notations, we will use the same symbols to design the associated functors on families of filtrations. 
Lemma 3.13. Let $\mathcal{E}$ be an equivariant reflexive sheaf on $X$. Assume that $\mathcal{E}=$ $\mathfrak{K}(\mathbb{E})$, with $\mathbb{E}=\left\{\left(E^{F}(i)\right) \subset E: F \prec P, i \in \mathbb{Z}\right\}$. Then the restriction $\mathcal{E}^{s}:=\iota^{*} \mathcal{E}$ is an equivariant reflexive sheaf on $X^{s}$ defined by the family of filtrations $\iota^{*} \mathbb{E}=$ $\left\{\left(E^{F}(i)\right) \subset E: F \prec P^{s}, i \in \mathbb{Z}\right\}$.

Proof. The proof follows from the fact that if $Q \prec P^{s}$, then for every facet $F$ that contains $Q, F \prec P^{s}$. Indeed, if $F$ contains $Q$, it intersects $U$ and thus lies in $P^{s s}=P^{s}$. Thus by definition of $\iota^{*} \mathbb{E}$, and from equation (4), we obtain the result.

Lemma 3.14. Let $\mathcal{E}$ be an equivariant reflexive sheaf on $X$. Assume that $\mathcal{E}=\mathfrak{K}(\mathbb{E})$, with $\mathbb{E}=\left\{\left(E^{F}(i)\right) \subset E: F \prec P, i \in \mathbb{Z}\right\}$. Then the invariant pushforward $\pi_{*}^{G} \mathcal{E}^{s}$ is an equivariant reflexive sheaf on $Y$ defined by the family of filtrations $\pi_{*}^{G} \mathbb{E}^{s}=$ $\left\{\left(\check{E}^{\check{F}}(i)\right) \subset \check{E}: \check{F} \prec P_{Y}, i \in \mathbb{Z}\right\}$, where

- $\check{E}=E$,

- $\check{E}^{\check{F}}(i)=E^{F}\left(b_{F} i\right)$ for all $F \prec P^{s}$, where we set $\check{F}=F \cap U$.

Proof. Let $F \prec P^{s}$. Then we have the GIT quotient projection

$$
\pi: U_{\sigma_{F}}=\operatorname{Spec}\left(\mathbb{C}\left[\sigma_{F}^{\vee} \cap M\right]\right) \rightarrow U_{\sigma_{F \cap U}}=\operatorname{Spec}\left(\mathbb{C}\left[\sigma_{U \cap F}^{\vee} \cap N_{0}^{\perp}\right]\right)
$$

and by definition,

$$
\Gamma\left(U_{\sigma_{F \cap U}}, \pi_{*}^{G} \mathcal{E}^{s}\right)=\bigoplus_{m \in N_{0}^{\perp}} \Gamma\left(U_{\sigma_{F}}, \mathcal{E}^{s}\right)_{m}
$$

Thus for all $m \in N_{0}^{\perp}$ :

$$
\Gamma\left(U_{\sigma_{F \cap U}}, \pi_{*}^{G} \mathcal{E}^{s}\right)_{m}=\Gamma\left(U_{\sigma_{F}}, \mathcal{E}^{s}\right)_{m}
$$

that is

$$
\check{E}^{U \cap F}\left(\left\langle m, \check{u}_{F}\right\rangle\right) \otimes \chi^{m}=E^{F}\left(\left\langle m, u_{F}\right\rangle\right) \otimes \chi^{m} .
$$

Using Lemma 3.9 and the basis $\check{\mathcal{B}}_{F}^{*}$ and $\mathcal{B}_{F}^{*}$ to decompose $m$ we obtain the result.

Similarly, we have

Lemma 3.15. Let $\check{\mathcal{E}}$ be an equivariant reflexive sheaf on $Y$. Assume that $\check{\mathcal{E}}=\mathfrak{K}(\check{\mathbb{E}})$, with $\check{\mathbb{E}}=\left\{\left(\check{E}^{\check{F}}(i)\right) \subset \check{E}: \check{F} \prec P_{Y}, i \in \mathbb{Z}\right\}$. Then the pull-back $\pi^{*} \check{\mathcal{E}}$ is an equivariant reflexive sheaf on $X^{s}$ defined by the family of filtrations $\pi^{*} \check{\mathbb{E}}=\left\{\left(E^{F}(i)\right) \subset E: F \prec\right.$ $\left.P^{s}, i \in \mathbb{Z}\right\}$ where

- $E=\check{E}$,

$$
\text { - } E^{F}(i)=\check{E}^{F \cap U}\left(\left\lfloor\frac{i}{b_{F}}\right\rfloor\right) \text { for all } F \prec P^{s} \text {. }
$$

In particular, the dimension jump values $e^{F}(i)$ can only be non-zero for values of $i$ that are multiples of $b_{F}$.

Proof. By Lemma 3.14, we know that $E^{F}\left(b_{F} i\right)=\check{E}^{U \cap F}(i)$ for $i \in \mathbb{Z}$, and hence we also have $E=\check{E}$. In general, we have for $j, i \in \mathbb{Z}$ :

$$
\check{E}^{U \cap F}(j) \otimes \chi^{j \check{m}_{F}}=\Gamma\left(U_{\sigma_{F \cap U}}, \check{\mathcal{E}}\right)_{j \check{m}_{F}}
$$

and

$$
E^{U \cap F}(i) \otimes \chi^{i m_{F}}=\Gamma\left(U_{\sigma_{F}}, \pi^{*} \check{\varepsilon}\right)_{i m_{F}} .
$$


Moreover, we have by definition

$$
\Gamma\left(U_{\sigma_{F}}, \pi^{*} \check{\mathcal{E}}\right)=\Gamma\left(U_{\sigma_{F \cap U}}, \check{\mathcal{E}}\right) \otimes_{\mathbb{C}\left[\sigma_{U \cap F}^{\vee} \cap N_{0}^{\perp}\right]} \mathbb{C}\left[\sigma_{F}^{\vee} \cap M\right] .
$$

Note that $m \in M \cap \sigma_{F}^{\vee}$ if and only if $\left\langle m, u_{F}\right\rangle \geq 0$ and $m \in N_{0}^{\perp} \cap \sigma_{U \cap F}^{\vee}$ if and only if $\left\langle m, \breve{u}_{F}\right\rangle \geq 0$. Thus, to prove the lemma, it is enough to show that if $i m_{F}=m^{\prime}+\left(i m_{F}-m^{\prime}\right)$ with $m^{\prime} \in N_{0}^{\perp} \cap \sigma_{U \cap F}^{\vee}$, and $\left(i m_{F}-m^{\prime}\right) \in M \cap \sigma_{F}^{\vee}$ then $\left\langle m^{\prime}, \check{u}_{F}\right\rangle \leq\left\lfloor\frac{i}{b_{F}}\right\rfloor$ (note that we use the fact that the spaces $\left(E^{F}(i)\right)$ form a filtration). Suppose then that we have such a decomposition $i m_{F}=m^{\prime}+\left(i m_{F}-m^{\prime}\right)$ for $i m_{F}$. Without loss of generality, we can assume that $m^{\prime}=a \check{m}_{F}$ with $a=\left\langle m^{\prime}, \check{u}_{F}\right\rangle \in \mathbb{N}$. Then we have $\left\langle i m_{F}-a \check{m}_{F}, u_{F}\right\rangle \geq 0$ and thus by Lemma 3.9 we obtain $i-a b_{F} \geq 0$. The result follows.

From these lemmas we deduce a version of Nevins' criterion for descent of equivariant reflexive sheaves on toric varieties [19]:

Corollary 3.16. Let $\mathcal{E}=\mathfrak{K}(\mathbb{E})$ be an equivariant reflexive sheaf on $X$ with dimension jumps $\left(e^{F}(i)\right)$. Then $\mathcal{E}$ descends to $Y$ if and only if for all facets $F \prec P^{s}$, for all $i \in \mathbb{Z}$ such that $e^{F}(i) \neq 0, b_{F}$ divides $i$.

Proof. The proof follows from the fact that $\mathcal{E}$ descends if and only if

$$
\iota^{*} \mathbb{E} \simeq \pi^{*} \pi_{*}^{G} \iota^{*} \mathbb{E} .
$$

Using Lemmas 3.14 and 3.15, this is equivalent that for all $F \prec P^{s}$, for all $i \in \mathbb{Z}$,

$$
E^{F}(i)=E^{F}\left(b_{F}\left\lfloor\frac{i}{b_{F}}\right\rfloor\right) .
$$

The result follows.

For later use, we emphasize the following corollary:

Corollary 3.17. Assume that $\mathcal{E}$ is an equivariant reflexive sheaf on $X$ that descends to $Y$, with $\mathcal{E}=\mathfrak{K}(\mathbb{E})$. Then for all $W \subset E$, the subsheaf $\mathcal{E}_{W} \subset \mathcal{E}$ descends to $Y$.

3.3. Pullback functors. We preserve notation of the previous section, still assuming the pair $(G, \gamma)$ to be generic. In this section, we introduce pull-back functors from $\mathfrak{R e} \mathfrak{f}^{T}(Y)$ to $\mathfrak{R} \mathfrak{e} \mathfrak{f}^{T}(X)$. Note that the superscript $T$ stands for equivariant, and means $T_{X}$-equivariant on $X$, and $T_{Y}$-equivariant on $Y$. The images of these functors will contain precisely the equivariant reflexive sheaves on $X$ that descend to $Y$ and that are suitable to compare slope stability notions on $X$ and $Y$.

Let $\mathbf{D}^{u}:=\left\{D_{F}: F \prec P^{u s}\right\}$ be the set of unstable irreducible $T$-equivariant divisors on $X$. For $\mathbf{i}:=\left(i_{D}\right)_{D \in \mathbf{D}^{u}} \in \mathbb{Z}^{u}$, we define a functor $\mathfrak{P}_{\mathbf{i}}$ from $\mathfrak{R e f} \mathfrak{f}^{T}(Y)$ to $\mathfrak{R e f}^{T}(X)$. Using the functor $\mathfrak{K}$, it is sufficient to define the corresponding functor from $\mathfrak{F i l t}\left(P_{Y}\right)$ to $\mathfrak{F i l t}(P)$. Let $\check{\mathbb{E}} \in \mathfrak{F} \mathfrak{i l t}\left(P_{Y}\right)$, with $\check{\mathbb{E}}=\left\{\left(\check{E}^{\check{F}}(i)\right) \subset \check{E}: \check{F} \prec P_{Y}, i \in\right.$ $\mathbb{Z}\}$. Then we define $\mathfrak{P}_{\mathbf{i}}(\check{\mathbb{E}})=\left\{\left(E^{F}(i)\right) \subset E: F \prec P, i \in \mathbb{Z}\right\}$ by setting $E=\check{E}$ and for $F \prec P$ and $i \in \mathbb{Z}$ :

- If $F \prec P^{s}$, then $E^{F}(i)=\check{E}^{U \cap F}\left(\left\lfloor\frac{i}{b_{F}}\right\rfloor\right)$.

- If $F \prec P^{u s}$, then

$$
E^{F}(i)=\left\{\begin{array}{lll}
0 & \text { if } & i<i_{D_{F}} \\
E & \text { if } \quad i \geq i_{D_{F}}
\end{array}\right.
$$


We now define the functors $\mathfrak{P}_{\mathbf{i}}$ on morphisms of families of filtrations. Let $\check{\mathbb{E}}_{1}, \check{\mathbb{E}}_{2} \in$ $\mathfrak{F i l t}\left(P_{Y}\right)$, with $\check{\mathbb{E}}_{j}=\left\{\left(\check{E}_{j}^{\check{F}}(i)\right) \subset \check{E}_{j}: \check{F} \prec P_{Y}, i \in \mathbb{Z}\right\}$, for $j \in\{1,2\}$. Let $\check{f} \in$ $\operatorname{Hom}\left(\check{\mathbb{E}}_{1}, \check{\mathbb{E}}_{2}\right)$, and recall that $\check{f}$ corresponds to a linear map $\check{f}: \check{E}_{1} \rightarrow \check{E}_{2}$ such that for all $\breve{F} \prec P_{Y}$ and $i \in \mathbb{Z}, \check{f}\left(\breve{E}_{1}^{\breve{F}}(i)\right) \subset \breve{E}_{2}^{\breve{F}}(i)$. We then simply define $\mathfrak{P}_{\mathbf{i}}(\check{f}):=f$ to be the element of $\operatorname{Hom}\left(\mathfrak{P}_{\mathbf{i}}\left(\check{\mathbb{E}}_{1}\right), \mathfrak{P}_{\mathbf{i}}\left(\check{\mathbb{E}}_{2}\right)\right)$ given by the linear map

$$
f=\check{f}: E_{1}=\check{E}_{1} \rightarrow E_{2}=\check{E}_{2} .
$$

We then check that $\mathfrak{P}_{\mathbf{i}}(\check{f})$ is well defined, that is for all $F \prec P$ and $i \in \mathbb{Z}$,

$$
f\left(E_{1}^{F}(i)\right) \subset E_{2}^{F}(i) .
$$

If $F \prec P^{u s}$, this is clear as for $i<i_{D_{F}}, E_{1}^{F}(i)=E_{2}^{F}(i)=0$, while for $i \geq i_{D_{F}}$, $E_{1}^{F}=E_{1}$ and $E_{2}^{F}=E_{2}$. If $F \prec P^{s}$, then $E_{j}^{F}(i)=\check{E}_{j}^{U \cap F}\left(\left\lfloor\frac{i}{b_{F}}\right\rfloor\right), j \in\{1,2\}$. However, by the compatibility of $\check{f}=f$ with the filtrations, for any $i^{\prime} \in \mathbb{Z}$, we have

$$
\check{f}\left(\check{E}_{1}^{U \cap F}\left(i^{\prime}\right)\right) \subset \check{E}_{2}^{U \cap F}\left(i^{\prime}\right),
$$

which implies $f\left(E_{1}^{F}(i)\right) \subset E_{2}^{F}(i)$. Thus $f$ is compatible with the families of filtrations and $\mathfrak{P}_{\mathbf{i}}(\check{f})$ is well defined.

By abuse of notation, we denote by $\mathfrak{P}_{\mathbf{i}}$ the associated functors on $\mathfrak{R e f}^{T}(Y)$. We then have:

Proposition 3.18. The functors $\mathfrak{P}_{\mathbf{i}}$ are fully faithful functors from the category $\mathfrak{R e f}^{T}(Y)$ to the category $\mathfrak{R e f}^{T}(X)$.

Proof. We keep the notations used to define $\mathfrak{P}_{\mathbf{i}}$. We need to show that the map

$$
\mathfrak{P}_{\mathbf{i}}: \operatorname{Hom}\left(\check{\mathbb{E}}_{1}, \check{\mathbb{E}}_{2}\right) \rightarrow \operatorname{Hom}\left(\mathfrak{P}_{\mathbf{i}}\left(\check{\mathbb{E}}_{1}\right), \mathfrak{P}_{\mathbf{i}}\left(\check{\mathbb{E}}_{2}\right)\right)
$$

is a bijection. Injectivity is clear by definition of $\mathfrak{P}_{\mathbf{i}}(\check{f})$. For surjectivity, let $f \in$ $\operatorname{Hom}\left(\mathfrak{P}_{\mathbf{i}}\left(\check{\mathbb{E}}_{1}\right), \mathfrak{P}_{\mathbf{i}}\left(\check{\mathbb{E}}_{2}\right)\right)$. Then $f$ is described by a linear map from $E_{1}$ to $E_{2}$ that is compatible with the families of filtrations $\mathfrak{P}_{\mathbf{i}}\left(\check{\mathbb{E}}_{1}\right)$ and $\mathfrak{P}_{\mathbf{i}}\left(\check{\mathbb{E}}_{2}\right)$. By definition of $\mathfrak{P}_{\mathbf{i}}$, for $F \prec P^{u s}$, the condition $f\left(E_{1}^{F}(i)\right) \subset E_{2}^{F}(i)$ is satisfied for any linear map from $E_{1}$ to $E_{2}$. However, for $F \prec P^{s}$, the compatibility gives for all $i \in \mathbb{Z}$ :

$$
f\left(\check{E}_{1}^{U \cap F}\left(\left\lfloor\frac{i}{b_{F}}\right\rfloor\right)\right) \subset \check{E}_{2}^{U \cap F}\left(\left\lfloor\frac{i}{b_{F}}\right\rfloor\right) .
$$

Taking in particular $i \in b_{F} \mathbb{Z}$, and using the fact that any facet of $P_{Y}$ is of the form $F \cap U$ for some facet $F \prec P^{s}$, we deduce that $f$ is a linear map between $\check{E}_{1}$ and $\check{E}_{2}$ that is compatible with the families of filtrations $\check{\mathbb{E}}_{1}$ and $\check{\mathbb{E}}_{2}$. Thus $f=\mathfrak{P}_{\mathbf{i}}(\check{f})$ for $\check{f}=f$, seen as a linear map from $\check{E}_{1}=E_{1}$ to $\check{E}_{2}=E_{2}$.

Remark 3.19. There is a simple relation between the different functors $\mathfrak{P}_{\mathbf{i}}$. Given an equivariant reflexive sheaf $\mathcal{E}$ on $Y$, for any $\mathbf{i}=\left(i_{D}\right)_{D \in \mathbf{D}^{u}} \in \mathbb{Z}^{u}$, one has

$$
\mathfrak{P}_{\mathbf{i}}(\mathcal{E})=\mathfrak{P}_{\mathbf{0}}(\mathcal{E}) \otimes \mathcal{O}_{X}\left(-\sum_{D \in \mathbf{D}^{u}} i_{D} D\right)
$$

As will appear clearly in the next section, the difference of slopes between two reflexive sheaves is not affected by tensoring both sheaves by $\mathcal{O}_{X}\left(-\sum_{D \in \mathbf{D}^{u}} i_{D} D\right)$. Thus, from the point of view of moduli spaces of slope stable sheaves, one is reduced to the study of the functor $\mathfrak{P}_{\mathbf{0}}$. We thank the referee for this interesting remark. 
Remark 3.20. The functors $\mathfrak{P}_{\mathrm{i}}$ might not preserve the compatibility conditions for local freeness introduced by Klyachko [12. We will give an explicit example of such phenomenon in Section 5.2 (Example 5.7). Note however that in the projective bundles examples (Section 5.3), $\mathfrak{P}_{0}$ is just a pullback and thus preserves local freeness.

We give a geometric interpretation of the images of the pull-back functors. First, we interpret the condition on unstable divisors with the following lemma, whose proof is straightforward from the definitions.

Lemma 3.21. Let $\mathcal{E}$ be an equivariant reflexive sheaf on $X$. Assume that $\mathcal{E}=\mathfrak{K}(\mathbb{E})$, with $\mathbb{E}=\left\{\left(E^{F}(i)\right) \subset E: F \prec P, i \in \mathbb{Z}\right\}$. Let $F$ be a facet of $P$ corresponding to an irreducible invariant divisor $D_{F}$ and let $i_{F} \in \mathbb{Z}$. Then the following are equivalent:

i) For all $i \in \mathbb{Z}$,

$$
E^{F}(i)=\left\{\begin{array}{lll}
0 & \text { if } \quad i<i_{F} \\
E & \text { if } \quad i \geq i_{F}
\end{array} .\right.
$$

ii) For all $m \in M$, a non-zero element of $\Gamma\left(U_{\sigma_{F}} \backslash O(F), \mathcal{E}\right)_{m}$ extends across the divisor $D_{F}$ to a section of $\Gamma\left(U_{\sigma_{F}}, \mathcal{E}\right)$ if and only if $\left\langle m, u_{F}\right\rangle \geq i_{F}$.

Remark 3.22. Condition (ii) in Lemma 3.21 is equivalent to the vanishing of the quantities $\left(\Delta_{j}(k)\right)$ introduced in [14, Proposition 3.20], for the index $j$ corresponding to $F$.

We deduce:

Corollary 3.23. Let $\mathcal{E}$ be an equivariant reflexive sheaf on $X$, and let $\mathbf{i} \in \mathbb{Z}^{u}$. Then the following are equivalent:

i) The sheaf $\mathcal{E}$ lies in the image of $\mathfrak{P}_{\mathbf{i}}$.

ii) The sheaf $\mathcal{E}$ descends to $Y$ and satisfies the extension condition (8) for all unstable divisor $D_{F}$, with $i_{F}=i_{D_{F}}$.

Proof. It follows from the definition of the functors and Lemmas 3.15 and 3.21

It will become clear in the next section that the extension condition (8) for unstable divisors is precisely the condition on $\mathfrak{P}_{\mathbf{i}}$ that enables us to compare the slopes of $\check{\varepsilon} \in \mathfrak{R e f}^{T}(Y)$ and of $\mathfrak{P}_{\mathbf{i}}(\check{\mathcal{E}})$ for all $\check{\mathcal{E}} \in \mathfrak{R e f}^{T}(Y)$. We finish this section with a more effective lemma that will be used in these comparisons:

Lemma 3.24. Let $\check{\mathcal{E}} \in \mathfrak{R e f}^{T}(Y)$ and $\mathcal{E}=\mathfrak{P}_{\mathbf{i}}(\check{\bar{\varepsilon}}) \in \mathfrak{R e f}^{T}(X)$. Let $\left(e^{F}(i)\right)$ be the dimension jumps of $\mathcal{E}$ and let $\left(\check{e}^{F}(i)\right)$ be the dimension jumps of $\check{\mathcal{E}}$. Then

i) For all $F \prec P^{s}, e^{F}(i)=0$ if $i \neq 0 \bmod b_{F}$ and $e^{F}\left(b_{F} i\right)=\check{e}^{F}(i)$.

ii) For all $F \prec P^{u s}, e^{F}(i)=0$ if $i \neq i_{D_{F}}$ and $e^{F}\left(i_{D_{F}}\right)=\operatorname{rk}(\mathcal{E})$.

Proof. It follows directly from the definition of the functors.

\section{Slopes under Descent and the Minkowski condition}

In this section, $(X, L)$ is a polarized toric variety with torus $T=N \otimes_{\mathbb{Z}} \mathbb{C}^{*}$, and $G=N_{0} \otimes_{\mathbb{Z}} \mathbb{C}^{*}$ is a subtorus. We assume $N_{0}=N \cap\left(N_{0} \otimes \mathbb{R}\right)$ and consider a linearization $\gamma$ such that $(G, \gamma)$ is generic (as in Definition 3.5). We will compare the slope stability notions on $X$ and $Y=X / / G$. We will use the same notation as in previous sections. 
4.1. Some stability notions. We recall now the notions of stabilities that we will consider, and state some propositions specific to the toric setting. We refer to [8,10] for the stability notions (see in particular [ 8 , Proposition 4.3] for the definition of slope on normal varieties) and to [15] for the definition of the intersection of a Weil divisor with real Cartier divisors.

Definition 4.1. Let $\mathcal{E}$ be a torsion-free coherent sheaf on $X$. The degree of $\mathcal{E}$ with respect to an ample class $\alpha \in N^{1}(X)_{\mathbb{R}}$ is the real number obtained by intersection:

$$
\operatorname{deg}_{\alpha}(\mathcal{E})=c_{1}(\mathcal{E}) \cdot \alpha^{n-1}
$$

and its slope with respect to $\alpha$ is given by

$$
\mu_{\alpha}(\mathcal{E})=\frac{\operatorname{deg}_{\alpha}(\mathcal{E})}{\operatorname{rk}(\mathcal{E})} .
$$

A torsion-free coherent sheaf $\mathcal{E}$ is said to be $\mu$-semi-stable or slope semi-stable with respect to $\alpha$ if for any coherent subsheaf $0 \neq \mathcal{F} \subsetneq \mathcal{E}$ with $0<\operatorname{rk} \mathcal{F}<\operatorname{rk} \mathcal{E}$, one has

$$
\mu_{\alpha}(\mathcal{F}) \leq \mu_{\alpha}(\mathcal{E}) .
$$

When strict inequality always holds, we say that $\mathcal{E}$ is $\mu$-stable. Finally, $\mathcal{E}$ is said to be $\mu$-polystable if it is the direct sum of $\mu$-stable subsheaves of the same slope.

It is a standard fact that to test stability of a torsion-free coherent sheaf $\mathcal{E}$, it is enough to check (9) for proper saturated coherent subsheaves $\mathcal{F}$ (see [10, Proposition 1.2.6]). A less standard fact that follows from [14, Proposition 4.13] is that in the toric setting, to check $\mu$-stability of an equivariant reflexive sheaf, it is enough to compare slopes for saturated equivariant reflexive subsheaves. More precisely, using the description of saturated equivariant reflexive subsheaves from Proposition 2.12, we have:

Proposition 4.2. 14, Proposition 4.13] and [9, Proposition 2.3]. Let $\mathcal{E}=\mathfrak{K}(\mathbb{E})$ be a $T$-equivariant reflexive sheaf on a normal toric variety $X$. Then $\mathcal{E}$ is $\mu$-semi-stable (resp. $\mu$-stable) if and only if for all proper vector subspaces $W \subset E, \mu_{L}\left(\mathcal{E}_{W}\right) \leq$ $\mu_{L}(\mathcal{E})$ (resp. $\mu_{L}\left(\mathcal{E}_{W}\right)<\mu_{L}(\mathcal{E})$ ), where $\mathcal{E}_{W}=\mathfrak{K}(W \cap \mathbb{E})$.

Note that the proof of the above proposition is valid on normal toric varieties. Following [9, 14], we will use the following combinatorial formula for the slopes of equivariant reflexive sheaves (see also [9, Lemma 2.2]):

Lemma 4.3. Let $\mathcal{E}$ be a $T$-equivariant reflexive sheaf with dimension jumps $\left(e^{F}(i)\right)$. Then

$$
\mu_{L}(\mathcal{E})=-\frac{1}{\operatorname{rk}(\mathcal{E})} \sum_{F \prec P} i_{F}(\operatorname{det} \mathcal{E}) \operatorname{deg}_{L}\left(D_{F}\right)
$$

where for all $F \prec P$,

$$
i_{F}(\operatorname{det} \mathcal{E})=\sum_{i \in \mathbb{Z}} i e^{F}(i) .
$$

Proof. It follows from the definition of slopes and Proposition 2.18,

We will need to compare the data defining the slopes after descent:

Lemma 4.4. Let $\mathbf{i}=\left(i_{F}\right)_{F \prec P u s} \in \mathbb{Z}^{u}$. Let $\check{\varepsilon}$ be a $T$-equivariant sheaf on $Y$. Then:

i) For all $F \prec P^{s}, i_{F}\left(\operatorname{det} \mathfrak{P}_{\mathbf{i}}(\check{\mathcal{E}})\right)=b_{F} i_{F \cap U}(\operatorname{det} \check{\mathcal{E}})$.

ii) For all $F \prec P^{u s}, i_{F}\left(\operatorname{det} \mathfrak{P}_{\mathbf{i}}(\tilde{\mathcal{E}})\right)=i_{F} \operatorname{rk}(\mathcal{E})$. 
Proof. The proof follows from the definition of $i_{F}(\operatorname{det} \mathcal{E})$ and Lemma 3.24

We will also need the degree for the pullbacks of the irreducible $T$-invariant divisors.

Lemma 4.5. Let $F_{1} \prec P^{s}$. Then for any $\mathbf{i} \in \mathbb{Z}^{u}$,

$$
\mathfrak{P}_{\mathbf{i}}\left(\mathcal{O}_{Y}\left(D_{F_{1} \cap U}\right)\right)=\mathcal{O}_{X}\left(b_{F_{1}} D_{F_{1}}-\sum_{F \prec P u s} i_{F} D_{F}\right) .
$$

Proof. The sheaf $\mathcal{O}_{Y}\left(D_{F_{1} \cap U}\right)$ is equivariant and reflexive. Combining Proposition 2.13 and the definition of the functor $\mathfrak{P}_{\mathbf{i}}, \mathfrak{P}_{\mathbf{i}}\left(\mathcal{O}_{Y}\left(D_{F_{1} \cap U}\right)\right)$ is determined by the family of filtrations

$$
\begin{aligned}
& L^{F_{1}}(i)= \begin{cases}0 & i<-b_{F_{1}}, \\
\mathbb{C} & i \geq-b_{F_{1}},\end{cases} \\
& L^{F}(i)=\left\{\begin{array}{ll}
0 & i<0, \\
\mathbb{C} & i \geq 0, \\
0 & i<i_{F}, \\
\mathbb{C} & i \geq i_{F},
\end{array} \text { if } F \neq F_{1}\right. \text { is stable, }
\end{aligned}
$$

That is to say, $\mathfrak{P}_{\mathbf{i}}\left(\mathcal{O}_{Y}\left(D_{F_{1} \cap U}\right)\right)=\mathcal{O}_{X}\left(b_{F_{1}} D_{F_{1}}-\sum_{F \prec P u s} i_{F} D_{F}\right)$.

Corollary 4.6. Let $F_{1} \prec P^{s}$. Then for any $\mathbf{i} \in \mathbb{Z}^{u}$,

$$
\operatorname{deg}_{L}\left(\mathfrak{P}_{\mathbf{i}}\left(\mathcal{O}_{Y}\left(D_{F_{1} \cap U}\right)\right)\right)=b_{F_{1}} \operatorname{deg}_{L}\left(D_{F_{1}}\right)-\sum_{F \prec P^{u s}} i_{F} \operatorname{deg}_{L}\left(D_{F}\right) .
$$

4.2. Comparison of slope stability via the Minkowski condition. In this section, we prove Theorem 4.7 and Proposition 4.8, from which Theorem 1.1 follows. Recall from Sections 2.1 and 3.1 that the data of $(X, L)$ together with the linearization $\gamma: T \rightarrow \operatorname{Aut}(L)$ determines a polytope $P \subset M_{\mathbb{R}}$, and that facets $F$ in the stable locus $P^{s}$ of $P$ correspond to stable irreducible $T$-invariant divisors $D_{F}$.

Theorem 4.7. Let $(X, L)$ be a polarized toric variety with torus $T=N \otimes_{\mathbb{Z}} \mathbb{C}^{*}$. Let $G=N_{0} \otimes_{\mathbb{Z}} \mathbb{C}^{*}$ be a subtorus for a saturated sublattice $N_{0} \subset N$. Let $\gamma: T \rightarrow \operatorname{Aut}(L)$ be a generic linearization of $G$, and denote by $Y$ the associated GIT quotient $X / / G$. Assume that Minkowski condition holds:

$$
\sum_{F \prec P^{s}} \operatorname{deg}_{L}\left(D_{F}\right) u_{F}=0 \quad \bmod N_{0} \otimes_{\mathbb{Z}} \mathbb{R} .
$$

Then there exists a unique ample class $\alpha \in N^{1}(Y)_{\mathbb{R}}$ such that for every equivariant reflexive sheaf $\check{\mathcal{E}}$ on $Y$, for any $\mathbf{i}=\left(i_{F}\right)_{F \prec P u s} \in \mathbb{Z}^{u}$, setting $\mathcal{E}=\mathfrak{P}_{\mathbf{i}}(\check{\mathcal{E}})$, we have

$$
\mu_{L}(\mathcal{E})=\mu_{\alpha}(\check{\mathcal{E}})-\sum_{F \prec P u s} i_{F} \operatorname{deg}_{L}\left(D_{F}\right) .
$$

In this case, $\mathcal{E}$ is stable on $(X, L)$ if and only if $\check{\mathcal{E}}$ is stable on $(Y, \alpha)$.

A converse of this statement is given in the following.

Proposition 4.8. There exists an ample class $\alpha \in N^{1}(Y)_{\mathbb{R}}$ with respect to which the functors $\mathfrak{P}_{\mathbf{i}}$ from equivariant reflexive sheaves on $Y$ to equivariant reflexive 
sheaves on $X$ preserve each of the conditions of $\mu$-stability, $\mu$-semi-stability and $\mu$-polystability if and only if

$$
\sum_{F \prec P^{s}} \operatorname{deg}_{P}\left(D_{F}\right) u_{F}=0 \bmod N_{0} \otimes_{\mathbb{Z}} \mathbb{R} .
$$

We delay the proofs slightly in order to first present the classical Minkowski theorem. We recall that a lattice $M$ defines a measure $\nu$ on $M_{\mathbb{R}}=M \otimes_{\mathbb{Z}} \mathbb{R}$ as the pull-back of Haar measure on $M_{\mathbb{R}} / M$. It is determined by the properties

(a) $\nu$ is translation invariant,

(b) $\nu\left(M_{\mathbb{R}} / M\right)=1$.

Let $\alpha \in N^{1}(X)_{\mathbb{R}}$ be an ample class, and denote by $P_{\alpha}$ the associated polytope. For any facet $F$ of $P_{\alpha}$, there is a unique $(n-1)$-form $\nu_{F}$, independent of $\alpha$, such that $\nu_{F} \wedge u_{F}=\nu$. If $P_{\alpha}$ is a lattice polytope, with

$$
P_{\alpha}=\left\{m \in M_{\mathbb{R}}:\left\langle m, u_{F}\right\rangle \geq-a_{F} \text { for all } F \prec P\right\},
$$

the form $\nu_{F}$ corresponds to the measure defined by the lattice

$$
M \cap\left\{m \in M_{\mathbb{R}}:\left\langle m, u_{F}\right\rangle=-a_{F}\right\}
$$

in the affine span of $F$. We will denote the volume of the facet $F$ with respect to $\nu_{F}$ by $\operatorname{latvol}(F)$.

Proposition 4.9 ( [3]). For any facet $F$ of $P_{\alpha}$,

$$
\operatorname{deg}_{\alpha}\left(D_{F}\right)=\operatorname{latvol}(F) .
$$

Proof. From [3, Section 11], the equality holds for $\alpha$ integral. Since the two expressions in the equality are both homogeneous (of order $n-1$ ) and continuous, we also have $\operatorname{deg}_{\alpha}\left(D_{F}\right)=\operatorname{latvol}(F)$, whenever $\alpha$ is an ample $\mathbb{R}$-divisor.

We have the elementary lemma:

Lemma 4.10. Let $u_{F} \in N$ be the primitive element associated to a facet $F \prec P$. Let $g=\langle\cdot, \cdot\rangle$ be a positive definite inner product on $M_{\mathbb{R}}$ whose induced volume form coincides with $\nu$ and let $\tilde{u}_{F}=u_{F} /\left\|u_{F}\right\|$ be the normalized vector in $N_{\mathbb{R}}$. Then,

$$
\operatorname{latvol}(F) u_{F}=\operatorname{vol}_{g}(F) \tilde{u}_{F} .
$$

Here $\operatorname{vol}_{g}$ denotes the $(n-1)$-dimensional Euclidean volume of the facet $F$, calculated with respect to the inner product $g$. From this observation we obtain the counterpart of a classical result of Minkowski, adapted to the case of lattice polytopes.

Proposition 4.11. Let $u_{1}, \ldots, u_{r} \in N$ be distinct primitive lattice elements that span the real vector space $N_{\mathbb{R}}$ and let $f_{1}, \ldots, f_{r}>0$ be positive real numbers. Then there exists a compact and convex polytope in $M_{\mathbb{R}}$ whose facets have inward normals the elements $\left(u_{i}\right)$ and lattice volumes the $\left(f_{i}\right)$ if and only if

$$
\sum_{i=1}^{r} f_{i} u_{i}=0 .
$$

Moreover, such a polytope is unique up to translation.

Proof. This follows immediately from the classical result (see [21, p.455]) and the previous lemma. 
We deduce the following interesting corollary on intersection theory of toric varieties:

Corollary 4.12. Let $\left(\alpha_{\rho}\right)_{\rho \in \Sigma(1)} \in \mathbb{R}_{>0}^{\sharp \Sigma(1)}$. For any ample class $\alpha \in N^{1}(X)_{\mathbb{R}}$ denote by $P_{\alpha}$ the associated polytope. Then the following two statements are equivalent:

i) There exists an ample class $\alpha \in N^{1}(X)_{\mathbb{R}}$ such that for all facets $F \prec P_{\alpha}$, $\operatorname{deg}_{\alpha}\left(D_{F}\right)=\alpha_{\rho_{F}}$,

ii) The following condition is satisfied:

$$
\sum_{F \prec P} \alpha_{\rho_{F}} u_{F}=0 .
$$

We can now give the proofs of Theorem 4.7 and Proposition 4.8 .

Proof of Theorem 4.7. Let $\mathbf{i} \in \mathbb{Z}^{u}$. Let $\check{\varepsilon}$ be an equivariant reflexive sheaf on $Y$ and let $\mathcal{E}=\mathfrak{P}_{\mathbf{i}}(\check{\mathcal{E}})$ on $X$, both of rank $r$. Let $\alpha$ be an ample $\mathbb{R}$-divisor on $Y$ that determines a real polytope $\check{P} \subseteq \check{M}_{\mathbb{R}}=N_{0}^{\perp} \otimes_{\mathbb{Z}} \mathbb{R}$ whose facets $\check{F}$ have primitive normals $\check{u}_{F}=u_{F \cap U} \in N / N_{0}$ equal to those of $P_{Y}$. Then, from Lemma 4.3,

$$
\mu_{\alpha}(\check{\mathcal{E}})=-\frac{1}{r} \sum_{\check{F} \prec \check{P}} i_{\breve{F}}(\operatorname{det} \check{\mathcal{E}}) \operatorname{deg}_{\alpha}\left(D_{\check{F}}\right)
$$

By the genericity assumption on $(G, \gamma)$, from Lemma 3.6, we deduce a bijective correspondence $F \leftrightarrow \check{F}$ between facets of $P^{s}$ and facets of $\check{P}$. Thus

$$
\mu_{\alpha}(\check{\mathcal{E}})=-\frac{1}{r} \sum_{F \prec P^{s}} i_{\check{F}}(\operatorname{det} \check{\mathcal{E}}) \operatorname{deg}_{\alpha}\left(D_{\check{F}}\right),
$$

whereas by Lemma 4.4 .

$$
\begin{aligned}
\mu_{L}\left(\mathfrak{P}_{\mathbf{i}}(\check{\mathcal{E}})\right) & =\mu_{L}(\mathcal{E}) \\
& =-\frac{1}{r} \sum_{F \prec P} i_{F}(\operatorname{det} \mathcal{E}) \operatorname{deg}_{L}\left(D_{F}\right), \\
& =-\frac{1}{r} \sum_{F \prec P^{s}} i_{\check{F}}(\operatorname{det} \check{\mathcal{E}}) b_{F} \operatorname{deg}_{L}\left(D_{F}\right)-\sum_{F \prec P^{u s}} i_{F} \operatorname{deg}_{L}\left(D_{F}\right) .
\end{aligned}
$$

Consider the right hand term of the last equality. We note two points:

(1) The second term $\sum_{F \prec P u s} i_{F} \operatorname{deg}_{L}\left(D_{F}\right)$ is independent of the reflexive sheaf $\check{\varepsilon}$. It depends only on the lattice volumes of the unstable facets $F \prec P^{u s}$ and on the indices $i_{F}$ that determine the pull-back functors.

(2) The first term coincides with $\mu_{\alpha}(\check{\mathcal{E}})$ if for all $F \prec P^{s}, \operatorname{deg}_{\alpha}\left(D_{\breve{F}}\right)=b_{F} \operatorname{deg}_{L}\left(D_{F}\right)$, which, by Proposition 4.9, is equivalent to latvol $(\check{\mathrm{F}})=b_{F} \operatorname{deg}_{L}\left(D_{F}\right)$.

By Proposition 4.11, we can chose the polytope $\check{P} \subseteq \check{M}_{\mathbb{R}}$ such that its facets satisfy $\operatorname{latvol}(\tilde{F})=b_{F} \operatorname{deg}_{L}\left(D_{F}\right)$ if and only if

$$
\sum_{\check{F} \prec \check{P}} b_{F} \operatorname{deg}_{L}\left(D_{F}\right) \check{u}_{F}=0,
$$

which, by Lemma 3.8 , is to say

$$
\sum_{F \prec P^{s}} \operatorname{deg}_{L}\left(D_{F}\right) u_{F}=0 \quad \bmod N_{0} \otimes_{\mathbb{Z}} \mathbb{R} .
$$

Thus, the Minkowski condition implies the existence of the desired polytope and of $\alpha$. 
Applying now (11) to the sheaf $\mathcal{O}\left(D_{\breve{F}}\right)$, using Corollary 4.6 we deduce that $\operatorname{deg}_{\alpha} D_{\breve{F}}=b_{F} \operatorname{deg}_{L}\left(D_{F}\right)$ for all $\check{F} \subset \check{P}$. Then, the uniqueness statement of Theorem 4.7 follows from the equality latvol $(\check{F})=\operatorname{deg}_{\alpha}\left(D_{\breve{F}}\right)$ and unicity in Proposition 4.11

As noted above in Proposition 4.2 (see [4,9]), to test for stability, it is sufficient to consider equivariant reflexive subsheaves of the form $\check{\mathcal{E}}_{W}=\mathfrak{K}(\mathbb{E} \cap W)$ where $W$ runs through vector subspaces of $E$. The slopes then satisfy

$$
\mu_{L}(\mathcal{E})-\mu_{L}\left(\mathcal{E}_{W}\right)=\mu_{\alpha}(\check{\mathcal{E}})-\mu_{\alpha}\left(\check{\mathcal{E}}_{W}\right)
$$

from which the equivalence of the stability conditions follows.

Proof of Proposition 4.8. The "if" statement is given in the previous theorem. We suppose that for some ample $\mathbb{R}$-divisor $\alpha$ on $Y$ the various stability notions are preserved by the pull-back functors. The ample divisor $\alpha$ defines a real polytope $\check{P} \subseteq \check{M}_{\mathbb{R}}=N_{0}^{\perp} \otimes_{\mathbb{Z}} \mathbb{R}$ whose facets $\check{F}$ have primitive normals $\check{u}_{F}=u_{F \cap U} \in N / N_{0}$.

We consider the functor $\mathfrak{P}_{\mathrm{i}}$ with $i_{F}=0$ for all unstable facets $F \prec P^{u s}$. For any two $F_{1}, F_{2} \prec P^{s}$ consider the direct sum of rank one reflexive sheaves $\mathcal{O}_{Y}\left(d_{1} D_{\check{F}_{1}}\right) \oplus$ $\mathcal{O}_{Y}\left(d_{2} D_{\breve{F}_{2}}\right)$ where $d_{1}=b_{F_{2}} \operatorname{deg}_{L}\left(D_{F_{2}}\right)$ and $d_{2}=b_{F_{1}} \operatorname{deg}_{L}\left(D_{F_{1}}\right)$. Then, using Lemma 4.5 ,

$$
\mathfrak{P}_{\mathbf{i}}\left(\mathcal{O}_{Y}\left(d_{1} D_{\breve{F}_{1}}\right) \oplus \mathcal{O}_{Y}\left(d_{2} D_{\breve{F}_{2}}\right)\right)=\mathcal{O}_{X}\left(d_{1} b_{F_{1}} D_{F_{1}}\right) \oplus \mathcal{O}_{X}\left(d_{2} b_{F_{2}} D_{F_{2}}\right)
$$

is the direct sum of rank-one reflexive sheaves of the same slope. By hypothesis, the initial sheaf must also be $\mu$-polystable so for any two stable facets of $P$,

$$
\operatorname{deg}_{\alpha}\left(b_{F_{2}} \operatorname{deg}_{L}\left(D_{F_{2}}\right) D_{\breve{F}_{1}}\right)=\operatorname{deg}_{\alpha}\left(b_{F_{1}} \operatorname{deg}_{L}\left(D_{F_{1}}\right) D_{\breve{F}_{2}}\right)
$$

so the expression

$$
c=b_{F} \frac{\operatorname{deg}_{L}\left(D_{F}\right)}{\operatorname{deg}_{\alpha}\left(D_{\breve{F}}\right)}
$$

is independent of $F \prec P^{s}$. Then, $\operatorname{latvol}(\check{F})=\operatorname{deg}_{\alpha}\left(D_{\check{F}}\right)$ and by the lattice Minkowski theorem (Proposition 4.11),

$$
\sum_{F \prec P^{s}} \operatorname{deg}_{\alpha}\left(D_{\check{F}}\right) \check{u}_{F}=0
$$

That is, by Lemma 3.8 ,

$$
\frac{1}{c} \sum_{F \prec P^{s}} b_{F} \operatorname{deg}_{L}\left(D_{F}\right) b_{F}^{-1} \pi\left(u_{F}\right)=0
$$

as desired.

\section{Applications}

In this section, we investigate the Minkowski condition. We show that any $n$ dimensional polarized toric orbifold $(X, L)$ admits at least $n+1$ GIT quotients by $\mathbb{C}^{*}$-actions that satisfy the Minkowski condition. The associated quotients are weighted projective spaces. We also consider the case of projectivization of torus invariant bundles. 
5.1. Compatible one parameter subgroups. Let $(X, L)$ be a polarized projective toric variety of complex dimension $n$ with torus $T=N \otimes_{\mathbb{Z}} \mathbb{C}^{*}$ and fan $\Sigma$. When considering subtori $G \subset T$, we will always assume that $G=N_{0} \otimes_{\mathbb{Z}} \mathbb{C}^{*}$, for a saturated sublattice $N_{0}$.

Definition 5.1. A subtorus $G \subset T$ is compatible with $(X, L)$ if there is a linearization $\gamma$ of $T$ on $L$ such that $(G, \gamma)$ is generic and $((X, L),(G, \gamma))$ satisfies Minkowski condition (10).

We will see that the Minkowski condition is very restrictive, in the sense that in most cases, for fixed $(X, L)$, there are few compatible one dimensional subtori. To see this, set $\operatorname{Div}_{\text {ir }}^{T}(X)=\left\{D_{\rho}, \rho \in \Sigma(1)\right\}$ be the set of reduced and irreducible $T$-invariant divisors on $X$. Let $\mathbf{D} \subset \operatorname{Div}_{\text {ir }}^{T}(X)$ be a non empty subset. Assume that there is a one parameter subgroup $G=N_{0} \otimes_{\mathbb{Z}} \mathbb{C}^{*} \subset T$ and a linearization $\gamma$ for $T$ on $L$ such that $(G, \gamma)$ is generic and the set of stable divisors for $(G, \gamma)$ is $\mathbf{D}$. Then, if Minkowski condition is satisfied, we have

$$
\sum_{D_{\rho} \in \mathbf{D}} \operatorname{deg}_{L}\left(D_{\rho}\right) u_{\rho}=0 \quad \bmod N_{0} \otimes_{\mathbb{Z}} \mathbb{R}
$$

Consider the element of $N$ :

$$
u_{\mathbf{D}}:=\sum_{D_{\rho} \in \mathbf{D}} \operatorname{deg}_{L}\left(D_{\rho}\right) u_{\rho} .
$$

Assume that $u_{\mathbf{D}}$ is not zero. Then Minkowski condition implies that

$$
N_{0}=N \cap\left(\mathbb{R} \cdot u_{\mathbf{D}}\right),
$$

so that in particular $G$ is entirely determined by $u_{\mathbf{D}}$ and thus by the set $\mathbf{D}$. So for a given $\mathbf{D}$ with $u_{\mathbf{D}}$ non-zero, there is at most one compatible one-parameter subgroup of $T$ with $\mathbf{D}$ as set of stable divisors. As the number of stable divisors is the number of faces of a given polytope in $M_{\mathbb{R}}$ that intersects the hyperplane $N_{0}^{\perp} \otimes_{\mathbb{Z}} \mathbb{R}$, in the generic situation, we must have $\sharp \mathbf{D} \in\{n, \ldots, \sharp \Sigma(1)-1\}$. Thus we have proved:

Lemma 5.2. Let $(X, L)$ be a $n$-dimensional projective polarized toric variety with fan $\Sigma$. Set $d=\sharp \Sigma(1)$ the number of rays of $\Sigma$. Assume that for all subset $\mathbf{D} \varsubsetneqq \operatorname{Div}_{\text {ir }}^{T}(X)$ with $\sharp \mathbf{D} \in\{n, \ldots, d-1\}$, we have $u_{\mathbf{D}} \neq 0$. Then, the number of one parameter subgroups compatible with $(X, L)$ is bounded by $\sum_{k=n}^{d-1}\left(\begin{array}{l}d \\ k\end{array}\right)$.

For example, the conditions of Lemma 5.2 are satisfied by Hirzebruch surfaces $\mathbb{F}_{a}$ for $a \geq 2$, but not by $\mathbb{P}^{1} \times \mathbb{P}^{1}$.

Remark 5.3. Note that if $X$ is a projective toric orbifold, then from the sequence

$$
0 \rightarrow M \rightarrow \bigoplus_{\rho \in \Sigma(1)} \mathbb{Z} \cdot D_{\rho} \rightarrow \mathrm{Cl}(X) \rightarrow 0
$$

and from the fact that the Picard group as finite index in the class group $\mathrm{Cl}(X)$, we deduce that $\sharp \Sigma(1)=n+p$, where $p$ is the Picard rank of $X$ (see [2, Theorem 4.1.3 and Proposition 4.2.7]). This gives the bound $\sum_{k=n}^{n+p-1}\left(\begin{array}{c}n+p \\ k\end{array}\right)$ on the number of compatible one-parameter subgroups. In particular, for the complex projective space $\mathbb{C P}^{n}$, this bound equals $n+1$. Using Proposition 5.4, we see that for a given dimension, this bound is achieved. 
5.2. Quotients to weighted projective spaces. In this section we will show:

Proposition 5.4. Let $(X, L)$ be a n-dimensional polarized toric orbifold with torus $T$ and associated polytope $P$ and denote by $m$ the number of torus fixed points of $X$. Then, up to replacing $L$ by a sufficiently high power, there exist at least $m$ one-parameter subgroups of $T$ compatible with $(X, L)$ giving distinct GIT quotients. The associated GIT quotients are weighted projective spaces.

From this proposition, we deduce that one can obtain $\mu$-stable reflexive sheaves on any polarized toric orbifold from $\mu$-stable reflexive sheaves on weighted projective spaces.

Remark 5.5. As weighted projective spaces have Picard rank 1, the number of rays of their fans, and thus the number of facets of their polytopes, is the smallest possible for a given dimension (see Remark 5.3 ). Thus, testing stability for reflexive sheaves is simpler on weighted projective spaces. We expect that these varieties could serve as simple bricks to study moduli spaces of equivariant reflexive sheaves on toric orbifolds, and will investigate in this direction in future work.

Proof. The argument is local at a vertex $v \in P$. As $X$ is an orbifold, its fan is simplicial, and thus the set $\left\{u_{F}, v \in F\right\}$ is a $\mathbb{Q}$-basis of $M_{\mathbb{Q}}$. Consider

$$
\mathbf{D}_{v}:=\left\{D_{F}: v \in F\right\}
$$

and

$$
u_{\mathbf{D}_{v}}=\sum_{D_{F} \in \mathbf{D}_{v}} \operatorname{deg}_{L}\left(D_{F}\right) u_{F}=\sum_{v \in F} \operatorname{deg}_{L}\left(D_{F}\right) u_{F} .
$$

As all the degrees $\operatorname{deg}_{L}\left(D_{F}\right)$ are positive, it is clear that $u_{\mathbf{D}_{v}}$ is not zero. Let $N_{0}=N \cap\left(\mathbb{R} \cdot u_{\mathbf{D}_{v}}\right)$ and let $G=N_{0} \otimes_{\mathbb{Z}} \mathbb{C}^{*}$. Then, by construction of $u_{\mathbf{D}_{v}}$, up to dilation and translations of $P$, we can assure that $P$ intersects $N_{0}^{\perp}$ transversally and precisely along the faces $F$ that contains $v$. Thus, up to scaling $L$ and choosing an appropriate linearization, the set of stable divisors for the $G$ action is $\mathbf{D}_{v}$. By construction, Minkowski condition is satisfied and $G$ is compatible with $(X, L)$. The associated quotient is a weighted projective space as the number of stable facets is $n$, giving $n$ rays for the $(n-1)$-dimensional toric GIT quotient. To conclude, the vertices in the polytope correspond to 0-dimensional orbits, hence to fixed points. Thus, there are at least $m$ such quotients, hence the result.

Remark 5.6. From Lemma 5.2 and Proposition 5.4 we deduce that the compatible one parameter subgroups for $\left(\mathbb{C P}^{n}, \mathcal{O}_{\mathbb{P} n}(1)\right)$ are given by the $\mathbb{C}^{*}$-actions

$$
\lambda \cdot\left[z_{0}, \ldots, z_{n}\right]=\left[z_{0}, \ldots, \lambda z_{i}, \ldots, z_{n}\right], i \in\{0, \ldots, n\},
$$

and the associated quotients are all isomorphic to $\mathbb{C P}^{n-1}$.

Example 5.7. Let $X=\mathbb{C P}^{1} \times \mathbb{C P}^{1} \times \mathbb{C P}^{1}$ together with the polarization $L=$ $\pi_{1}^{*} \mathcal{O}_{\mathbb{P}^{1}}(4) \otimes \pi_{2}^{*} \mathcal{O}_{\mathbb{P}^{1}}(4) \otimes \pi_{3}^{*} \mathcal{O}_{\mathbb{P}^{1}}(4)$ where $\pi_{j}: X \rightarrow \mathbb{C P}^{1}$ denotes the projection onto the $j$-th factor. The associated polytope $P$ in $\mathbb{Z}^{3} \otimes_{\mathbb{Z}} \mathbb{R}=\mathbb{R}^{3}$ is a cube with edges of length 4 . Up to translation, that is up to changing the linearisation of the torus action on $L$, we can assume that $P$ is

$$
P=[-3,1] \times[-3,1] \times[-3,1] \subset \mathbb{R}^{3} .
$$


Consider now the one parameter subgroup $G$ of $T$ whose lattice is $N_{0}=\mathbb{Z} \cdot(1,1,1)$. The GIT quotient of $(X, L)$ by $G$ is the toric variety $Y$ associated to the polytope $U \cap P=(1,1,1)^{\perp} \cap P$, that is

$$
P_{Y}=\left\{(x, y, z) \in \mathbb{R}^{3}: x+y+z=0, x, y, z \in[-3,1]\right\} .
$$

We recognize the polytope of $\mathbb{C P}^{2}$, so that $Y \simeq \mathbb{C P}^{2}$. The stable facets in $P^{s}$ are given by $F_{x}=\{x=1\} \cap P, F_{y}=\{y=1\} \cap P$ and $F_{z}=\{z=1\} \cap P$. The degree of the associated invariant divisors $D_{x}, D_{y}$ and $D_{z}$ is equal to 16 with respect to $L$, so the Minkowski condition becomes

$$
16 u_{D_{x}}+16 u_{D_{y}}+16 u_{D_{z}} \in N_{0} \otimes_{\mathbb{Z}} \mathbb{R}=\mathbb{R} \cdot(1,1,1) .
$$

As $u_{D_{x}}=(-1,0,0), u_{D_{y}}=(0,-1,0)$ and $u_{D_{z}}=(0,0,-1)$, the Minkowski condition is satisfied. By Theorem 1.1, and from the fact that, up to scaling, the only polarization on $\mathbb{C P}^{2}$ is $\mathcal{O}_{\mathbb{P}^{2}}(1)$, an equivariant reflexive sheaf $\check{\mathcal{E}}$ on $\mathbb{C P}^{2}$ is slope stable with respect to $\mathcal{O}_{\mathbb{P}^{2}}(1)$ if and only if $\mathfrak{P}_{\mathbf{i}}(\check{\mathcal{E}})$ is slope stable on $X$ with respect to $L$. For example, the tangent sheaf $\mathcal{T}_{\mathbb{C P}^{2}}$ is slope stable with respect to $\mathcal{O}_{\mathbb{P}^{2}}(1)$ (see e.g. [9, Theorem 1.3]). Thus we obtain a slope stable rank 2 equivariant reflexive sheaf on $(X, L)$ by setting $\mathcal{E}=\mathfrak{P}_{\mathbf{0}}\left(\mathcal{T}_{\mathbb{C P}^{2}}\right)$. Note that this equivariant sheaf is not locally free, and hence $\mathfrak{P}_{\mathbf{0}}$ doesn't preserve local freeness in general. To see this, recall from [12] that the family of filtrations defining $\mathcal{T}_{\mathbb{C P}^{2}}$ is given by $\left\{\left(T^{\check{F}}(i)\right) \subset N_{Y} \otimes_{\mathbb{Z}} \mathbb{C}: \check{F} \prec P_{Y}, i \in \mathbb{Z}\right\}$ where

$$
T^{\check{F}}(i)=\left\{\begin{array}{ccc}
0 & \text { if } & i \leq-2 \\
\operatorname{Vect}\left(\check{u}_{F}\right) & \text { if } & i=-1 \\
N_{Y} \otimes_{\mathbb{Z}} \mathbb{C} & \text { if } & i \geq 0 .
\end{array}\right.
$$

We compute $b_{F_{x}}=b_{F_{y}}=b_{F_{z}}=1$ here, so that the family of filtrations associated to $\mathcal{E}$ is $\mathbb{E}=\left\{\left(E^{F}(i)\right) \subset E: F \prec P, i \in \mathbb{Z}\right\}$, where in particular $E^{F}(i)=T^{F \cap U}(i)$ for $F \in\left\{F_{x}, F_{y}, F_{z}\right\}$. Klyachko's criterion for $\mathcal{E}$ to be locally free imposes a condition on the family of filtrations $\mathbb{E}$ for each face of $P$ (see e.g. [20, Theorem 5.22] for the statement with increasing filtrations). In particular, for the vertex $(1,1,1) \in P$, this condition states that there should be a $\mathbb{Z}^{3}$-grading of $E=N_{Y} \otimes_{\mathbb{Z}} \mathbb{C}$ :

$$
E=\bigoplus_{m \in \mathbb{Z}^{3}} E_{m}
$$

such that for each face $F \prec P$ containing $(1,1,1)$, one has

$$
E^{F}(i)=\sum_{\left\langle m, u_{F}\right\rangle \leq i} E_{m}
$$

As for such faces, $\check{u}_{F}=\pi\left(u_{F}\right)$, where $\pi: \mathbb{Z}^{3} \rightarrow N_{Y}=\mathbb{Z}^{3} / \mathbb{Z} \cdot(1,1,1)$ denotes the projection map, and $E^{F}(i)=T^{U \cap F}(i)$, we deduce that we would have

$$
E_{(1,0,0)}=\operatorname{Vect}\left(\check{u}_{F_{x}}\right), E_{(0,1,0)}=\operatorname{Vect}\left(\check{u}_{F_{y}}\right), E_{(0,0,1)}=\operatorname{Vect}\left(\check{u}_{F_{z}}\right) \text {. }
$$

But then $\operatorname{dim}(E) \geq 3$, which is absurd as $\operatorname{dim}(E)=\operatorname{rk}\left(N_{Y}\right)=2$. Hence $\mathbb{E}$ doesn't satisfy Klyachko's criterion and $\mathcal{E}$ is not locally free.

5.3. Projectivization of toric vector bundles. As a motivating example, we consider the variety given as the projectivization of a torus invariant vector bundle. We refer to [15, Appendix A] and [2, Section 7.3] for the construction and classical results. Let $Y$ be a projective toric variety with fan $\Sigma_{Y}$. For $i=1, \ldots, r$, let 
$D_{i}=\sum_{\rho \in \Sigma_{Y}(1)} a_{i \rho} D_{\rho}$ be invariant divisors on $Y$ and let $V_{\mathcal{F}}$ be the vector bundle associated to the locally free sheaf

$$
\mathcal{F}=\mathcal{O}_{Y} \oplus \mathcal{O}_{Y}\left(D_{1}\right) \oplus \cdots \oplus \mathcal{O}_{Y}\left(D_{r}\right) .
$$

Let $X=\mathbb{P}\left(V_{\mathcal{F}}^{\vee}\right)$ be the projectivization of the dual of $V_{\mathcal{F}}$, with $\pi: X \rightarrow Y$ the projection to the base $Y$. Then $X$ is a projective toric variety, with torus $T_{X}=$ $T_{Y} \times\left(\mathbb{C}^{*}\right)^{r}$. Denote by $N_{X}, N_{Y}$ the lattices of $X$ and $Y$, and by $M_{X}$ and $M_{Y}$ their duals. Then $N_{X}=N_{Y} \times \mathbb{Z}^{r}$ and we have exact sequences

$$
\begin{gathered}
0 \longrightarrow \mathbb{Z}^{r} \longrightarrow N_{Y} \times \mathbb{Z}^{r} \longrightarrow N_{Y} \longrightarrow 0, \\
0 \longrightarrow M_{Y} \longrightarrow M_{Y} \times\left(\mathbb{Z}^{r}\right)^{*} \longrightarrow\left(\mathbb{Z}^{r}\right)^{*} \longrightarrow 0 .
\end{gathered}
$$

Let $L_{Y}$ be an ample line bundle on $Y$ and let $\mathcal{O}_{X}(1)=\mathcal{O}_{\mathbb{P}\left(V_{\mathcal{F}}\right)}(1)$ be the Serre line bundle on $X$. Then, up to replacing $L_{Y}$ by a sufficiently high power, $L_{X}=$ $\pi^{*} L_{Y} \otimes \mathcal{O}_{X}(1)$ is an ample line bundle on $X$. Assume now that $L_{Y}=\mathcal{O}\left(D_{Y}\right)$ for an ample divisor on $Y$

$$
D_{Y}=\sum_{\rho \in \Sigma_{Y}(1)} b_{\rho} D_{\rho}
$$

with associated lattice polytope

$$
P_{Y}=\left\{m \in\left(M_{Y}\right)_{\mathbb{R}}:\left\langle m, u_{\rho}\right\rangle \geq-b_{\rho} \text { for all } \rho \in \Sigma_{Y}(1)\right\} .
$$

To determine a polytope $P_{X} \subseteq\left(M_{Y}\right)_{\mathbb{R}} \times \mathbb{R}^{r}$ associated to $\left(X, L_{X}\right)$, we first describe the invariant irreducible divisors of $X$. They are of two types:

$$
\operatorname{Div}_{i r r}^{T}(X)=\left\{\pi^{-1}\left(D_{\rho}\right): \rho \in \Sigma_{Y}(1)\right\} \cup\left\{\left\{s_{i}=0\right\} \text { for } i=0,1, \ldots, r\right\}
$$

where the $\left\{s_{i}=0\right\}$ are the relative hyperplane sections associated to the line subbundles of $V_{\mathcal{E}}^{\vee}$. Set $\hat{D}_{\rho}=\pi^{-1}\left(D_{\rho}\right)$ for all $\rho \in \Sigma_{Y}(1)$ and $D_{s_{i}}:=\left\{s_{i}=0\right\}$ for all $i \in\{0, \ldots, r\}$. Then $L_{X}=\mathcal{O}(\hat{D})$ with

$$
\hat{D}=\sum_{\rho \in \Sigma_{Y}(1)} b_{\rho} \hat{D}_{\rho}+\sum_{i=0}^{r} D_{s_{i}}
$$

and associated polytope $P_{X}$ defined by $\left(m_{y}, m_{x}\right) \in\left(M_{Y}\right)_{\mathbb{R}} \times\left(\mathbb{R}^{r}\right)^{*}$ is in $P_{X}$ if and only if

$$
\begin{array}{r}
\left\langle m_{x}, e_{i}\right\rangle \geq-1, \text { for all } i=0, \ldots, r, \text { and } \\
\left\langle\left(m_{y}, m_{x}\right), u_{\rho}+\sum_{i=1}^{r} a_{i \rho} e_{i}\right\rangle \geq-b_{\rho}, \text { for all } \rho \in \Sigma_{Y}(1),
\end{array}
$$

where $\left\{e_{i}: i=1, \ldots, r\right\}$ is the standard basis of $\mathbb{R}^{r}$ and $e_{0}=-\left(e_{1}+\ldots+e_{r}\right)$.

We will perform a GIT quotient of $X$ by the torus $G=N_{0} \otimes_{\mathbb{Z}} \mathbb{C}^{*}$, where $N_{0}=$ $\left\{0_{Y}\right\} \times \mathbb{Z}^{r}$. Consider the linearization $\gamma_{y}$ for $T_{Y}$ on $L_{Y}$ giving the polytope $P_{Y}$ (recall Section 3.1, equation (7D). Consider the standard linearization $\gamma_{x}$ of $\left(\mathbb{C}^{*}\right)^{r}$ on $\mathcal{O}_{\mathbb{C P}^{r}}(1)$ associated to the polytope

$$
\left\{\left\langle m_{x}, e_{i}\right\rangle \geq-1, \text { for all } i=0, \ldots, r\right\} \subset\left(\mathbb{R}^{r}\right)^{*} .
$$

Combining the two gives the linearization $\gamma=\gamma_{y} \times \gamma_{x}$ of $T_{X}$ on $L_{X}$ associated to $P_{X}$. Then, setting $U=N_{0}^{\perp} \otimes_{\mathbb{Z}} \mathbb{R}$, we have $U=\left(M_{Y}\right)_{\mathbb{R}} \times\{0\}$. In particular, the only facets of $P_{X}$ that intersect $U$ are those given by equations $\left\langle m_{y}, u_{\rho}\right\rangle+\sum_{i} a_{i \rho}\left\langle m_{x}, e_{i}\right\rangle=-b_{\rho}$ for some $\rho \in \Sigma_{Y}(1)$ and the polytope $P_{X} \cap U$ is $P_{Y} \times\{0\}$. That is, the GIT quotient of $\left(X, L_{X}\right)$ by $G$ is $\left(Y, L_{Y}\right)$. We also note that $(G, \gamma)$ is generic. 
Proposition 5.8. The polarized toric variety $\left(X, L_{X}\right)$, with toric subgroup $G=$ $\left(\mathbb{C}^{*}\right)^{r} \subseteq T_{X}$ and linearization $\gamma$ satisfies the Minkowski condition of Theorem 4.7.

Proof. The line bundle $L_{X}$ determines a polytope in $\left(M_{X}\right)_{\mathbb{R}}$ whose normal fan equals the fan of $X$. By Corollary 4.12, we see that

$$
\sum_{F \prec P_{X}} \operatorname{deg}_{L_{X}}\left(D_{F}\right) u_{F}=0,
$$

and so

$$
\sum_{F \prec P_{X}^{s}} \operatorname{deg}_{L_{X}}\left(D_{F}\right) u_{F}=-\sum_{F \prec P_{X}^{u s}} \operatorname{deg}_{L_{X}}\left(D_{F}\right) u_{F} .
$$

Note that the unstable divisors are precisely the $D_{s_{i}}$ for $i \in\{0, \ldots r\}$. Moreover, the normal elements $u_{F}$, for $F \prec P_{X}^{u s}$, are given by the basis elements $e_{i} \in \mathbb{R}^{r}$ together with $e_{0}$. Then, $\pi\left(e_{i}\right)=0 \in N_{Y}=N_{X} / \mathbb{Z}^{r}$ and hence

$$
\sum_{F \prec P_{X}^{s}} \operatorname{deg}_{L_{X}}\left(D_{F}\right) u_{F}=0 \quad \bmod N_{0} \otimes_{\mathbb{Z}} \mathbb{R}
$$

as desired.

The GIT quotient map $p: X^{s} \rightarrow Y$ coincides with the restriction $\left.\pi\right|_{X^{s}}$ so it makes sense to compare two methods of pulling back sheaves from $Y$ to $X$. Given a $T$-equivariant reflexive sheaf $\mathcal{E}$ on $Y$, for each $\mathbf{i} \in \mathbb{Z}^{r+1}$, we have invariant reflexive sheaves $\pi^{*} \mathcal{E}$ and $\mathfrak{P}_{\mathbf{i}}(\mathcal{E})$ on $X$.

Proposition 5.9. Taking $\mathbf{i}=0$, if $\check{\varepsilon}$ is a T-equivariant reflexive sheaf on $Y$, we have

$$
\pi^{*} \check{\mathcal{E}}=\mathfrak{P}_{0}(\check{\mathcal{E}}) \text {. }
$$

Proof. Suppose that $\check{\mathcal{E}}=\mathfrak{K}(\check{\mathbb{E}})$ where $\check{\mathbb{E}}=\left\{\left(E^{\check{F}}(i)\right) \subset E: \check{F} \prec P_{Y}, i \in \mathbb{Z}\right\}$. Then, $\pi^{*} \check{\mathcal{E}}$ and $\mathfrak{P}_{0}(\check{\mathcal{E}})$ are determined up to isomorphism by their respective filtrations. On one hand, $\mathfrak{P}_{0}(\check{\mathbb{E}})=\left\{\left(E^{F}(i)\right) \subset E: F \prec P_{X}, i \in \mathbb{Z}\right\}$ is given by the filtration

$$
E^{F}(i)=\left\{\begin{array}{ccc}
E^{F \cap U}(i) & \text { for } & F \prec P^{s}, i \in \mathbb{Z}, \\
0 & \text { for } & F \prec P^{u s}, i<0, \\
E & \text { for } & F \prec P^{u s}, i \geq 0 .
\end{array}\right.
$$

On the other hand, by Lemma 3.15, for all $F \prec P_{X}^{s}$, observing that $b_{F}=1$, we have $\left(\pi^{*} \check{\mathbb{E}}\right)^{F}(i)=E^{F \cap U}(i)$. Let $F_{i} \prec P_{X}^{u s}$ be the unstable facet corresponding to $D_{s_{i}}$. The morphism $\pi: X \rightarrow Y$ corresponds to a lattice homomorphism $\bar{\pi}: N_{X} \rightarrow N_{Y}$. From the fact that $\bar{\pi}$ sends the normal generator $u_{F_{i}}=e_{i}$ of the ray $\rho_{F_{i}}$ to $0 \in N_{Y}$, we deduce that the image of the affine chart $\pi\left(U_{\sigma_{F_{i}}}\right)$ is $T_{Y} \subseteq Y$. Hence,

$$
\Gamma\left(U_{\sigma_{F_{i}}}, \pi^{*} \check{\varepsilon}\right)=\Gamma\left(T_{Y}, \check{\varepsilon}\right) \otimes_{\mathbb{C}\left[M_{Y}\right]} \mathbb{C}\left[\rho_{F_{i}}^{\vee} \cap M_{X}\right]=E \otimes \mathbb{C}\left[\rho_{F_{i}}^{\vee} \cap M_{X}\right]
$$

from which it follows that

$$
\left(\pi^{*} \check{\mathbb{E}}\right)^{F_{i}}(j)= \begin{cases}0 & \text { for } j<0 \\ E & \text { for } j \geq 0\end{cases}
$$

allowing us to conclude that $\pi^{*} \mathcal{E}=\mathfrak{P}_{0}(\mathcal{E})$.

From Propositions 5.8 and 5.9, together with Theorem 4.7 we obtain 
Proposition 5.10. There exists an ample class $\alpha$ on $Y$ such that an equivariant reflexive sheaf $\mathcal{E}$ on $Y$ is $\mu$-stable with respect to $\alpha$ if and only if $\pi^{*} \mathcal{E}$ is $\mu$-stable on $X$ with respect to $L_{X}$.

We conclude with a special case where the ample class $\alpha$ can be computed explicitly, that is for $Y$ of dimension 2 and $V_{\mathcal{F}}$ of rank 3 . It seems unlikely that a similar method could be used to compute the ample class $\alpha$ for higher dimensions and rank. Nevertheless, the examples below show that in general, $\alpha$ is different from the polarization induced by the GIT quotient.

Lemma 5.11. With previous notations, assume in addition that $Y$ is an orbifold of dimension 2 and that $V_{\mathcal{F}}$ is of rank 3. Then the class $\alpha$ from Proposition 5.10 is equal to $c_{1}\left(L_{Y}^{3} \otimes \operatorname{det}\left(V_{\mathcal{F}}\right)\right)$, up to scale.

Proof. As in that setting $Y$ and $X$ are orbifolds, we can make use of Poincaré duality to compute intersections, and degrees. From the proof of Theorem 4.7, we see that $\alpha$ is the ample class that satisfies for all $\rho \in \Sigma_{Y}(1)$,

$$
\operatorname{deg}_{\alpha}\left(D_{\rho}\right)=\operatorname{deg}_{L_{X}}\left(\hat{D}_{\rho}\right) .
$$

We compute the later using that $H^{2}(X, \mathbb{Z})$ is the algebra over $H^{2}(Y, \mathbb{Z})$ generated by the class $\xi:=c_{1}\left(\mathcal{O}_{X}(1)\right)$, with relation $\xi^{3}=\pi^{*} c_{1}\left(V_{\mathcal{F}}\right) \cdot \xi^{2}-\pi^{*} c_{2}\left(V_{\mathcal{F}}\right) \cdot \xi+\pi^{*} c_{3}\left(V_{\mathcal{F}}\right)$. Taking into account the dimensions, and the rank of $V_{\mathcal{F}}$, a direct computation gives

$$
\operatorname{deg}_{L_{X}}\left(\hat{D}_{\rho}\right)=c_{1}\left(L_{X}\right)^{3} \cdot \pi^{*} c_{1}\left(\mathcal{O}\left(D_{\rho}\right)\right)=\pi^{*}\left(3 c_{1}\left(L_{Y}\right)+c_{1}\left(V_{\mathcal{F}}\right)\right) \cdot \pi^{*} c_{1}\left(\mathcal{O}\left(D_{\rho}\right)\right) \cdot \xi^{2} .
$$

The result follows from Whitney's formula.

\section{REFERENCES}

[1] Daniele Angella and Cristiano Spotti. Kähler-Einstein metrics: old and new. Complex Manifolds, 4(1):200-244, 2017.

[2] D. Cox, J. Little, and H. Schenck. Toric Varieties. Providence, RI: American Mathematical Society (AMS), 0 edition, 2011.

[3] V. I. Danilov. The geometry of toric varieties. Uspekhi Mat. Nauk, 33(2(200)):85-134, 247, 1978.

[4] J. Dasgupta, A. Dey, and B. Khan. Stability of equivariant vector bundles over toric varieties. ArXiv preprint 1910.13964.

[5] Simon Donaldson. Kähler-Einstein metrics and algebraic geometry. In Current developments in mathematics 2015, pages 1-25. Int. Press, Somerville, MA, 2016.

[6] Akito Futaki. The Ricci curvature of symplectic quotients of Fano manifolds. Tohoku Math. J. (2), 39(3):329-339, 1987.

[7] Oscar García-Prada. Invariant connections and vortices. Comm. Math. Phys., 156(3):527$546,1993$.

[8] Henri Guenancia. Semistability of the tangent sheaf of singular varieties. Algebr. Geom., 3(5):508-542, 2016.

[9] M. Hering, B. Nill, and H. Suess. Stability of tangent bundles on smooth toric Picard-rank-2 varieties and surfaces. ArXiv preprint 1910.08848.

[10] Daniel Huybrechts and Manfred Lehn. The Geometry of Moduli Spaces of Sheaves. Cambridge Mathematical Library. Cambridge University Press, 2 edition, 2010.

[11] Nathan Ilten and Hendrik Süss. Equivariant vector bundles on T-varieties. Transform. Groups, 20(4):1043-1073, 2015.

[12] A. A. Klyachko. Equivariant bundles over toric varieties. Izv. Akad. Nauk SSSR Ser. Mat., 53(5):1001-1039, 1135, 1989.

[13] Shoshichi Kobayashi. Differential geometry of complex vector bundles, volume 15 of Publications of the Mathematical Society of Japan. Princeton University Press, Princeton, NJ; Princeton University Press, Princeton, NJ, 1987. Kanô Memorial Lectures, 5. 
[14] Martijn Kool. Fixed point loci of moduli spaces of sheaves on toric varieties. Adv. Math., 227(4):1700-1755, 2011

[15] Robert Lazarsfeld. Positivity in algebraic geometry. I, volume 48 of Ergebnisse der Mathematik und ihrer Grenzgebiete. 3. Folge. A Series of Modern Surveys in Mathematics [Results in Mathematics and Related Areas. 3rd Series. A Series of Modern Surveys in Mathematics]. Springer-Verlag, Berlin, 2004. Classical setting: line bundles and linear series.

[16] V. B. Mehta and A. Ramanathan. Restriction of stable sheaves and representations of the fundamental group. Invent. Math., 77(1):163-172, 1984.

[17] D. Mumford, J. Fogarty, and F. Kirwan. Geometric invariant theory, volume 34 of Ergebnisse der Mathematik und ihrer Grenzgebiete (2) [Results in Mathematics and Related Areas (2)]. Springer-Verlag, Berlin, third edition, 1994.

[18] Ignasi Mundet i Riera. Parabolic vector bundles and equivariant vector bundles. Internat. J. Math., 13(9):907-957, 2002.

[19] Thomas Nevins. Descent of coherent sheaves and complexes to geometric invariant theory quotients. J. Algebra, 320(6):2481-2495, 2008.

[20] Markus Perling. Graded rings and equivariant sheaves on toric varieties. Math. Nachr., 263/264:181-197, 2004.

[21] Rolf Schneider. Convex Bodies: the Brunn-Minkowski Theory, volume 44 of Encyclopedia of Mathematics and its Applications. Cambridge University Press, 2nd. edition, 2013.

[22] Michael Thaddeus. Toric quotients and flips. In Topology, geometry and field theory, pages 193-213. World Sci. Publ., River Edge, NJ, 1994.

Instituto de Matemática, Universidade Federal do Rio de Janeiro, Av. Athos da Silveira Ramos 149, Rio de Janeiro, RJ, 21941-909, Brazil

E-mail addresses: andrew@im.ufrj.br

Univ Brest, UMR CNRS 6205, Laboratoire de Mathématiques de Bretagne AtlanTIQUe, France

E-mail addresses: carl.tipler@univ-brest.fr 